\title{
Lipid utilization in skeletal muscle cells is modulated in vitro and in vivo by
} specific miRNAs

Francesco Chemello ${ }^{1,2}$, Francesca Grespi ${ }^{1,3}$, Alessandra Zulian ${ }^{4}$, Pasqua Cancellara ${ }^{4}$, Etienne Hebert-Chatelain ${ }^{1,3}$, Paolo Martini ${ }^{1}$, Camilla Bean ${ }^{1,3}$, Enrico Alessio ${ }^{1,2}$, Ruggero Ferrazza ${ }^{5}$, Paolo Laveder ${ }^{1}$, Graziano Guella ${ }^{5}$, Carlo Reggiani ${ }^{4}$, Chiara Romualdi $^{1}$, Paolo Bernardi ${ }^{4}$, Luca Scorrano ${ }^{1,3}$, Stefano Cagnin ${ }^{1,2,6 \ddagger}$, and Gerolamo Lanfranchi ${ }^{1,2,6 \ddagger}$

1- Department of Biology, University of Padova, Via Ugo Bassi 58/b 35131 Padova, Italy

2- CRIBI Biotechnology Centre, University of Padova, Via Ugo Bassi 58/b 35131 Padova, Italy

3- Venetian Institute of Molecular Medicine, Via Orus 2, 35131 Padova, Italy

4- Department of Biomedical Sciences, University of Padova, Via Ugo Bassi 58/b 35131 Padova, Italy

5- Department of Physics, University of Trento, Via Sommarive 14, 38123 Povo (Trento), Italy

6- CIR-Myo Myology Center, University of Padova, Via Ugo Bassi 58/b 35131 Padova, Italy

† Corresponding Authors: Gerolamo Lanfranchi, gerolamo.lanfranchi@unipd.it; Stefano Cagnin, stefano.cagnin@unipd.it 


\section{SUMMARY}

Skeletal muscle is composed by different myofiber types that can preferentially use glycolysis or lipids for ATP production. How fuel preference is specified in these post-mitotic cells is unknown. Here we show that miRNAs are important players in defining the myofiber metabolic profile. mRNA and miRNA signatures of all myofiber types obtained at single cell level unveiled fiber-specific regulatory networks and identified two master miRNAs that coordinately control myofiber fuel preference and mitochondrial morphology. Our work provides a complete and integrated myofiber type-specific catalogue of genes and miRNAs expressed and establishes miR-27a-3p and miR-142-3p as key regulators of lipid utilization in skeletal muscle.

\section{KEYWORDS}

Single myofiber, skeletal muscle metabolism, mitochondria, miRNAs, lipids.

\section{HIGHLIGHTS}

- Transcriptional networking in single cells distinguished myofibers based on glycolytic or oxidative metabolism, regulated by specific miRNAs

- $\quad$ miR-27a-3p and -142-3p influence mitochondrial morphology

- $\quad$ miR-27a-3p improves lipid utilization and increases glycogen storage both in vitro and in vivo

- $\quad$ miR-142-3p reduces lipid utilization both in vitro and in vivo 


\section{INTRODUCTION}

Half of the body mass of healthy individuals is constituted by skeletal muscle that supports body movements and is crucial in intermediate metabolism. These anatomical and physiological features are shared throughout the Vertebrate phylum, where muscle exerts a chief role in regulation of whole body metabolism. As a metabolic organ, skeletal muscle regulates systemic glucose homeostasis. When glucose is not consumed as fuel, it is accumulated as glycogen by muscle, and this storage may be almost five times more efficient as in the liver (Jensen et al., 2011). However, during physiological changes such as, for example, fasting, muscle can rapidly adapt to use fatty acids (FAs) and amino acids as oxidative substrates. Not surprisingly, impairment in muscle metabolic plasticity and insulin sensitivity is a hallmark of several metabolic diseases including type II diabetes and obesity (Sears and Perry, 2015).

The flexibility of skeletal muscle in the utilization of carbohydrate or lipid is associated with the expression of different panels of structural proteins that ultimately define different contractile properties (Schiaffino and Reggiani, 2011). The overall physiological diversity and plasticity is based on the repertoire and proportion of different types of myofibers that compose a particular muscle in a particular physiological condition. In small mammals, slow muscles such as soleus are mostly composed of slow-oxidative myofibers that express the slow type 1 myosin heavy chain (MyHC) and fast-oxidative myofibers that express type 2A or 2X MyHC. Type 1, 2A, and $2 \mathrm{X}$ mouse myofibers are considered oxidative myofibers and prevalently utilize lipids to meet most of their energy requirements aerobically. Instead, fast muscles, such as extensor digitorum longus (EDL), gastrocnemius, and the tibialis anterior, are mostly composed by fast-glycolytic myofibers that express a 
predominance of type 2B MyHC and utilize glucose to produce most of energy anaerobically. Mixed with these four major types of myofibers, minor intermediate hybrid fibers expressing different combinations of MyHC isoforms can be found in different muscles.

Metabolic adaptation can also occur through the modulation of mitochondrial dynamics (Liesa and Shirihai, 2013; Schrepfer and Scorrano, 2016). In skeletal muscle, mitochondrial dynamics is tailored to the different myofiber types: mitochondria are elongated due to increased fusion in oxidative myofibers compared to glycolytic myofibers (Mishra et al., 2015). This layer of regulation can contribute to explain how muscle, a terminally differentiated and post-mitotic tissue, can rapidly adapt its metabolism.

Muscle adaptive capacity to specific physiological stimuli should rely on complex, ordered changes in the pattern of fiber gene expression. Indeed, gene expression profiles of muscles with different metabolic traits are different (Campbell et al., 2001; Wu et al., 2003) and sensor molecules exist that, upon specific stimuli, may act as transcriptional modifiers (Bean et al., 2008; Carrasco and Hidalgo, 2006). MicroRNAs (miRNAs) are small non-coding RNAs (20-22 nt long) that posttranscriptionally control gene expression and can modulate metabolism (Dumortier et al., 2013). miRNAs were also recently implicated in the regulation of mitochondrial metabolism in skeletal muscle. It was demonstrated that mice lacking miR-378-3p and $-5 \mathrm{p}$ encoded by the PGC1beta locus are resistant to obesity induced by high-fat diet and can efficiently oxidize FAs (Carrer et al., 2012). However, whether such a regulation occurs normally and is involved in the specification of the metabolic preference of individual fibers is unknown. 
To define networks of coding and non-coding RNAs that regulate skeletal muscle metabolism, a genomic approach in single isolated myofibers is required, to avoid the averaging effect of multiple fiber types and signals of non-muscle cells (Chemello et al., 2011; Chemello et al., 2015; Mammucari et al., 2015; Murgia et al., 2015). Here, we compiled a comprehensive catalogue of mRNAs and miRNAs expressed in single myofiber types of mouse muscles. This allowed us to obtain the RNA regulatory networks supervising the metabolic phenotypes of myofibers and to identify two specific miRNAs (miR-27a-3p and miR-142-3p) that, by influencing mitochondrial shape and respiration, define lipid and glycogen utilization and hence the oxidative and glycolytic myofiber traits. 


\section{RESULTS}

\section{The transcriptional signature of a myofiber echoes its metabolic trait.}

We undertook a large-scale expression profiling of single isolated myofibers, by collecting 100 myofibers from soleus and 100 myofibers from EDL mouse muscles. In a fragment of each fiber, we measured MyHC isoforms as a reference to the canonical fiber catalogue (Figure 1A and S1). As expected, this method identified 6 major patterns of MyHC isoforms, namely 1, 2A, 2A/2X, $2 \mathrm{X}$ for soleus, and 2X/2B, 2B for EDL (Table S1). We therefore profiled 10 myofibers from each of the six major MyHC groups (Dataset S1), generating a collection of 60 transcriptional profiles. Totally, 1,936 probes resulted differentially expressed (DE) among the fiber repertoire (Dataset S2). Cluster analysis gathered the 60 profiles in a larger signature composed by 40 fibers and in a smaller one summing up the remaining 20 fibers (Figure 1B). The larger signature was clearly divided in two sub-signatures. One, that we named transcriptional slow (tS, 14 soleus fibers), was mainly composed by type 1 fibers and the other, called transcriptional intermediate (tI, 26 soleus fibers), was composed by type $2 \mathrm{~A}, 2 \mathrm{~A} / 2 \mathrm{X}$ and $2 \mathrm{X}$ fibers. The smaller signature, composed by type $2 \mathrm{~B}$ and $2 \mathrm{X} / 2 \mathrm{~B}$ fibers, was named transcriptional fast (tF, 20 fibers from EDL). To obtain a quick and univocal assay for the assignment of myofibers to one of the three signatures, we identified the 5 genes with the best classification power (Figure S2A), further including Myh2 transcript as the best marker for tI signature (Figure S2B and Dataset S3). As for MyHC proteins, at transcriptional level Myh mRNAs are excellent markers for myofiber typing (Figure S2C).

To understand the functional meaning of the gene clustering architecture, which divided the DE probes in 8 groups (Figure S2D and Dataset S4), we categorized 
them evidencing that the biological processes differing between the 3 myofiber signatures are principally related to cellular metabolism (Table 1 and Dataset S5). In fact, the two major clusters of fiber profiles reflected the two metabolic traits of skeletal muscle tissue, i.e. oxidative and glycolytic. tS and tI myofibers were enriched in genes coding for enzymes involved in FA, electron transport chain, and acetyl-CoA metabolic processes (Figure 1B); $\mathrm{tF}$ myofibers expressed preferentially genes coding for glucose metabolism proteins (Figure 1B). These experiments confirmed that different myofiber types display specific metabolic gene signatures. Interestingly, Gene Ontology analysis indicated that mitochondrial genes are up-regulated especially in tI myofibers, suggesting the high importance of the organelle in the metabolism of this type of myofibers.

\section{MicroRNAs post-transcriptionally regulate myofiber metabolism.}

miRNAs are important post-transcriptional regulators of cell metabolic pathways (Dumortier et al., 2013; Rottiers and Naar, 2012). We explored their role in the regulation of myofiber metabolic mRNA signatures. Populations of mRNAs and miRNAs were purified and separated from single isolated myofibers. The miRNA fractions were analysed by next-generation sequencing (NGS) in each fiber type, transcriptionally classified using the previously established mRNA markers (Figure S3). We identified 81 miRNAs expressed in $\mathrm{tS}, 84$ in $\mathrm{tI}$, and 75 in tF myofibers (Figure 1C and Dataset S6). We recognized 15 significantly enriched families, belonging to known muscle-specific miRNAs (myomiRs; (McCarthy, 2011), namely miR-1a, b/206/613, miR-133a, b, c and miR-486-5p/3107; Dataset S7). In association to miR-1a-3p and miR-133a-3p (which we confirmed as two of the most expressed miRNAs in muscle fibers) we identified 10 other miRNAs highly expressed in all 
fiber types (Table S2). These include almost all members of the let-7 family, indicating their importance in skeletal muscle biology. Interestingly, we could associate specific miRNAs to different transcriptional signatures (Figure S4A). For example, myomiR-206-3p and miR-208b-3p that control skeletal muscle oxidative metabolism (Boettger et al., 2014; Gan et al., 2013) are strictly expressed in tS myofibers. Quantitative RT-PCR measures of 55 miRNAs confirmed the NGS results with a correlation of 0.78 (Figure S4B and Dataset S8). Certain miRNA-3p/miRNA$5 \mathrm{p}$ couples were differentially expressed across different myofibers, as already evidenced in other tissue types (Martini et al., 2014).

The identification of miRNA-targets in an mRNA population is critical. For this reason, putative miRNA-targets were extracted from the repertoire of mRNAs expressed in muscle fibers by predictive algorithms and filtered for biological significance. As miRNAs act through target degradation (Hu and Coller, 2012), only miRNA-mRNA groups that showed a negative correlation between their expression levels were selected for the construction of an integrated network. A total of 5,968 interactions were computed, resulting from the negative correlations between the expression of 79 miRNAs and 1,051 putative mRNA targets (Dataset S9). The topology of the miRNA-mRNA interactions clearly draws two sub-networks where miRNAs are placed as central regulators (Figure 1D and Figure S5). The subnetwork 1 is mainly characterized by genes coding for mitochondrial proteins and enzymes involved in oxidative phosphorylation and FA metabolism, whereas the subnetwork 2 contained genes encoding for glycolytic enzymes. Considering the number of edges for each node in the network it follows that each miRNA, on average, contacts 81 mRNAs, supporting the idea that a single miRNA may regulate many mRNAs. Comparing the networks for $\mathrm{tS}$ and $\mathrm{tF}$ myofibers an opposite expression 
trend is clearly observed for connected genes and miRNAs (Figure 2D). In tS fibers, transcripts involved in glycolytic metabolism were generally under-expressed while the connected miRNAs were up-regulated; conversely, transcripts involved in oxidative phosphorylation and FA metabolism were up-regulated, while the connected miRNAs were down-regulated. The picture was specular in tF fibers. The network for tI fibers generally showed intermediate expression levels. In summary, this set of data provides the first cell-specific expression network that may control the glycolytic and oxidative metabolic traits.

miR-27a-3p and -142-3p influence mitochondrial shape and respiration in vitro.

Our data indicate that differences in mitochondrial genes characterize the myofiber signatures, particularly those of tI myofibers, (Figure 1B and Table 1), supporting the role of this organelle in directing metabolic plasticity of skeletal muscle (Hood et al., 2006). We selected for functional analyses a set of miRNAs that are connected though a different number of transcripts in the metabolic networks represented in Figure 1D. These are miR-27a-3p and -23a-3p, connected to 114 and 105 putative mRNA targets; and miR-130a-3p and -142-3p connected to 73 and 48 mRNA targets. In whole muscle analysis, all these miRNAs are more expressed in slow muscle, except for miR-142-3p that does not vary between fast and slow muscles (Figure 2A). NGS analysis on single myofibers revealed that miR-23a-3p and $-130 a-3 p$ were upregulated in $\mathrm{tS}$ and tI myofibers, miR-27a-3p was up-regulated in tI myofibers and, on the contrary, miR-142-3p was down-regulated in tI myofibers (Figure 2B). Since function and shape of mitochondria are strictly related (Schrepfer and Scorrano, 2016), we examined the role of the four selected miRNAs in mitochondrial morphology. In mouse muscle $\mathrm{C} 2 \mathrm{C} 12$ cells, the modification of miR-23a-3p or miR- 
130a-3p expression did not affect mitochondrial shape, while increased levels of miR27a-3p or miR-142-3p caused mitochondrial fragmentation (Figure 2, C and D). Electron microscopy confirmed that myoblasts transfected with these two miRNAs possess smaller and less-connected mitochondria, although with normal cristae structure (Figure S6A). The altered mitochondrial morphology was accompanied by changes in mitochondrial fuel oxidation. In myoblasts relying on glucose as oxidable substrate, miR-27a-3p caused a significant decrease in maximal oxygen consumption rate (OCR) induced by FCCP (Figure 2, E and F). When the oxidable substrate was palmitate, miR-142-3p decreased, whereas miR-27a-3p increased cellular respiration (Figure 2, G and H). Coherently, myoblasts over-expressing miR-142-3p showed mitochondrial fragmentation in palmitate medium and altered cristae structure, whereas no alteration in cristae structure was observed in myoblasts over-expressing miR-27a-3p (Figures 2I, S6A, and S6B). We investigated if the alteration of bioenergetics supply, observed in myoblasts transfected in vitro with miR-27a-3p or $142-3 p$, could result in accumulation of unused nutrients that eventually leads to the observed mitochondrial fragmentation, as already demonstrated in other experimental paradigms (Liesa and Shirihai, 2013; Schrepfer and Scorrano, 2016). miR-27a-3p induced the accumulation of glycogen (Figure 2J), whereas miR-142-3p that of lipid droplets in myoblasts (Figure $\mathbf{2 K}$ ), in the proximity of mitochondria (Figure S6C). To understand what type of lipids were accumulated in myoblasts transfected with miR-142-3p, we profiled them by lipidomics. This unbiased analysis distinguished 66 lipids, belonging to 10 different classes (Dataset S10) and confirmed an overall increase in lipid content after miR-142-3p over-expression (Figure S6D and S6E for the classes not significantly altered). Phosphatidylcholine (the major phospholipid populating the surface of lipid droplets) and triacylglycerol (the main constituent of 
the neutral lipid core of lipid droplets) are among the classes of lipids that increased

(Figure 2L), being probably the major components of miR-142-3p induced fat droplets.

These results indicate that miR-27a-3p and miR-142-3p act on cellular metabolic pathways inducing morphological and physiological dysfunction of mitochondria. FA utilization is inhibited by miR-142-3p, and it is improved by miR-27a-3p, whereas glycogen consumption is limited by over-expression of miR-27a-3p. Gene expression analyses of myoblasts over-expressing miR-27a-3p or miR-142-3p were coherent with these functional changes. In fact, miR-27a-3p depressed electron transport chain genes, whereas miR-142-3p turned off genes coding for enzymes involved in FA metabolism (Dataset S11).

miR-27a-3p and -142-3p influence metabolism of muscle cells by targeting Pgm2,

\section{Gaa and Fndc5.}

We next evaluated if miR-27a-3p and miR-142-3p down-regulated the putative targets identified by the integration of mRNA and miRNA myofibers expression signatures (Figure 2C). Microarray analysis showed that $66.7 \%$ and $73.9 \%$ of miR27a-3p or miR-142-3p targets were indeed down-regulated (Figure 3A). Interestingly, peroxisome proliferator-activated receptor $\gamma$ (Pparg), a validated target of miR-27a-3p (Kim et al., 2010) was down-regulated by 1.5 fold. Because miR-27a-3p influences glycogen utilization, we searched for genes involved in glycogenolysis in the list of putative targets down-regulated in $\mathrm{C} 2 \mathrm{C} 12$ cells over-expressing miR-27a-3p. Phosphoglucomutase (Pgm2) and acid $\alpha$-glucosidase (Gaa), genes encoding for key enzymes respectively in cytosolic and lysosomal glycogenolysis, were among the targets. The interaction between miR-27a-3p and the 3'-UTR of Pgm2 and Gaa was 
confirmed by luciferase assay (Figure 3B). In addition, glycogen phosphorylase (Pygm), another important gene for glycogenolysis not directly targeted by miR-27a$3 p$, was under-expressed when miR-27a-3p is up-regulated in C2C12 cells (Table S3).

In C2C12 myoblasts expressing miR-142-3p, fibronectin type III domain containing 5 (Fndc5), encoding for the precursor of the myokine Irisin (Bostrom et al., 2012) was the most down-regulated gene. To validate this negative connection, we transfected C2C12 myoblasts with a construct containing the 3'-UTR of Fndc5 driving the expression of luciferase, observing a $40 \%$ reduction of activity upon over-expression of miR-142-3p (Figure 3B). Transcriptomic analysis of myoblasts over-expressing miR-142-3p indicated that several genes involved in FA metabolism were deregulated. Acyl-CoA synthase 1 (Acsl1), the most important isoform for the synthesis of acyl-CoA used by skeletal muscle mitochondria (Li et al., 2015), was upregulated. This confirms an increase in FA anabolism, although other acyl-CoA synthase isoforms (Acsl3, Acs16) were found to be down-regulated. Genes of FA catabolic enzymes, like acyl-CoA dehydrogenase (Acad10, Acad12), or related to FA catabolism, like carnitine palmitoyl transferase (Cpt2), hormone sensitive lipase (HSL), and Ucp2 and 3 were down-regulated. Conversely, the lipid droplet markers perilipins (Plin1, Plin2, Plin4) were up-regulated (Table S3). These in vitro overexpression experiments suggest the principal mediators by which miR-27a-3p and miR-142-3p might exert their differential control of fuel consumption in muscle cells.

miR-27a-3p and -142-3p control fuel availability in vivo.

To test whether these two miRNAs can also induce glycogen or lipid accumulation in vivo, we transfected hindlimb mouse muscles with miRNA mimics. In muscle over- 
expressing miR-27a-3p, periodic acid-Schiff (PAS) staining confirmed glycogen accumulation (Figure 4, A and B). Also, electron microscopy analysis established the presence of abundant glycogen granules and showed that mitochondria are impaired, but generally maintaining regular cristae structure (Figure 4C). The expression of miR-142-3p in vivo recapitulated the results obtained in $\mathrm{C} 2 \mathrm{C} 12$ myoblasts, with stronger BODIPY staining when muscles were transfected with the miRNA mimic (Figure 4, D and E), and clearly visible lipid droplets in electron microscopy images (Figure 4D).

Interestingly, upon miR-27a-3p over-expression succinate dehydrogenase staining indicated an increase in oxidative myofibers and a reduction in glycolytic myofibers, in agreement with the over-expression of mRNAs for oxidative enzymes in tI myofibers. No such variations were observed instead in muscles transfected with miR-142-3p (Figure 4, F and G).

qRT-PCR experiments on transfected muscles confirmed the reduced expression of the same miRNA targets already established with the in vitro experiments (Figure 3C). Pgm2 and Gaa expression was inhibited by miR-27a-3p, whereas Fndc5 was down-regulated by miR-142-3p. Furthermore, other master genes involved in FA metabolism such as Cpt2, Plin2, and Lipe were down-regulated in muscles upon miR$142-3 p$ transfection.

In summary, all the results of in vitro and in vivo experiments agreed in showing the differential role of miR-27a-3p and $-142-3 p$ in the regulation of fuel consumption in skeletal muscle. 


\section{DISCUSSION}

In this work, we describe how the metabolic plasticity of myofibers relies on dynamic transcriptional networks that govern their fuel preferences and, by generating and browsing the compendium of mRNAs and miRNAs expressed in single isolated myofibers, we identify two miRNAs that can modulate the lipid utilization in myofibers.

Previous transcriptional catalogues obtained from whole muscles samples (Campbell et al., 2001; Raz et al., 2018; Wu et al., 2003) represent an average transcriptome of the different muscle cell repertoires, and lack the resolution power to identify the specific signatures of the different myofiber types. This is particularly important when inspecting the transcriptional signatures that dictate the metabolic traits. However, the evolution of single cell approaches allows the identification of signatures that specify functional and metabolic behaviour of the individual myofiber type. Our analysis of transcriptional signatures shows that the preferential metabolism (oxidative or glycolytic) is the key component that describes the myofiber molecular phenotype. tS and tI myofibers display high expression levels of genes encoding for enzymes of oxidative phosphorylation and FA oxidation, whereas tF myofibers preferentially express genes encoding for enzymes of glycolytic metabolism. This picture is closer to the more traditional functional classification of muscle myofibers, that differentiates them in slow-oxidative, fast-oxidative, and fast-glycolytic (Peter JB et al., 1972), than to the MyHC-based classification. Interestingly, we observed that tI myofibers (mostly composed by type 2A, 2X, and 2A/2X myofibers) are characterized by the highest level of expression of genes coding for mitochondrial enzymes. This observation agrees with previous proteomic analysis of single myofibers (Murgia M et al., 2015) that found an enrichment of mitochondrial proteins 
involved in oxidative phosphorylation in type $2 \mathrm{~A}$ fibers, in accordance with their largest content in mitochondria (Schiaffino S et al., 1970).

Microarrays and NGS are approaches particularly suitable to snapshot the transcriptome of single myofibers; yet, to profile non-coding RNAs and to assign them to a specific myofiber subtype, miRNA and mRNA expression profiles must be integrated. Such an approach identified post-transcriptional circuits involved in the regulation of myofiber metabolism, in addition to those already established involving miR-208b-3p, miR-499-5p and Sox6 (Gan et al., 2013; van Rooij et al., 2009). Our integrated approach highlights two previously unappreciated major circuits that connect metabolic transcripts with miRNAs $27 \mathrm{a}-3 \mathrm{p}$ and $142-3 \mathrm{p}$. miRNA profiling in whole muscle described these miRNAs as more expressed in slow muscle or not differentially expressed between fast and slow muscle, respectively. The single myofiber approach instead revealed their opposite expression in the tI myofibers, miR-27a-3p being highly, and miR-142-3p poorly expressed. This set of findings suggests a complementary effect of these two miRNAs in regulating myofiber metabolism. Functional assays in vitro and in vivo reveal that miR-27a-3p improves lipid utilization and reduces the breakdown of glycogen and consequently the glycolysis (Figure 5A). These adaptations in the selection of specific fuel type due to miR-27a-3p are coherent with the oxidative metabolism of tI myofibers, and explain the ability of the miRNA to change the myofiber phenotype from glycolytic to oxidative. On the contrary, miR-142-3p inhibits lipid utilization in myofibers (Figure 5A). However, also tS myofibers, which use lipids as fuel, express miR-142-3p suggesting the different repertoire of miRNA expression in tS and tI fibers can differently influence genes involved in lipid catabolism. 
Increased miRNAs dosage leads to accumulation of unused nutrients (i.e., glycogen and lipids) and to mitochondrial fragmentation, which appears to be due to fission. This event is likely to be secondary to the accumulation of reduced nutrients, as opposed to the elongation occurring upon nutrient starvation (Gomes et al., 2011; Rambold et al., 2011). While changes in morphology appear ancillary, mitochondrial fuel utilization is conversely embedded in the program controlled by these metabolic signatures. Expression of miR-27a-3p results in reduced maximal mitochondrial respiration, and in glycogen accumulation due to inhibition of cytosolic and lysosomal glycogen breakdown. Indeed, we found that miR-27a-3p expression represses Pgm2 (an enzyme involved in the conversion of glucose 1 phosphate generated by the glycogen phosphorylase into glucose 6 phosphate) and Gaa (a lysosomal enzyme essential for the hydrolysis of glycogen to glucose) (Figure 5B). Concomitantly, miR27a-3p improves lipids utilization in agreement with its established role in adipocytes and hepatocytes (Ji et al., 2009; Shirasaki et al., 2013; Wang et al., 2011).

miR-142-3p impairs FA utilization, resulting in the accumulation of lipid droplets. Indeed, miR-142-3p expression is lowest in tI myofibers, characterized by the highest expression of mitochondrial genes. Conversely, miR-142-3p over-expression shuts down the FA mitochondrial transporter carnitine palmitoyltransferase and the uncoupling proteins Ucp2 and Ucp3. Ucp3 prevents triglyceride storage (Musa et al., 2012) and its expression increases in C2C12 myoblasts exposed to the myokine irisin (Vaughan et al., 2014). Interestingly, the irisin precursor Fndc5 is the most downregulated among miR-142-3p targets (Figure 5B). miR-142-3p therefore emerges as a crucial molecule controlling the Fndc5/irisin pathway since it inhibits irisin autocrine effects, impairing FA utilization and eventually leading to lipid droplets accumulation. Indeed, miR-142-3p down-regulates HSL and up-regulates perilipins, whereas 
Fndc5/irisin promotes lipolysis exactly by triggering the opposite effect on HSL and perilipins (Xiong et al., 2015).

The miRNAs and targets discovered in our work are likely participants in obesity and diabetes. Indeed, a rat model of type 2 diabetes and mature adipocytes from obese mice display deregulation of miR-27a-3p as well as of its target Gpd2 (Herrera et al., 2010; Kim et al., 2010; MacDonald et al., 1996). miR-142-3p was associated with alterations in lipid pathways and abnormal fat deposition also in brain (Di Paolo and Kim, 2011) and it is a biomarker for morbidity of obesity and type 2 diabetes (Ortega et al., 2013; Ortega et al., 2014). We can envisage that a muscular metabolic reprogramming by delivery of miRNAs or antagomirs detailed in this work could be a promising route for the development of treatments counteracting the pathological side effects of diabetes and obesity. 


\section{ACKNOWLEDGMENTS}

Authors wish to thank dr. Federico Caicci (BioImaging Facility of the Department of Biology, University of Padova) for help with electron microscopy analyses; dr. Caterina Millino and dr. Beniamina Pacchioni (Microarray Service MicroCribi, CRIBI Biotechnology Centre, University of Padova), for the help with microarray analyses; Professor Fabio Di Lisa and dr. Valeria Petronilli for useful discussions. This work was supported by grants of the CARIPARO Foundation (FIBRE-GEXP) and of the University of Padova (CPDA139317) to S.C., Cariplo foundation (MAYBE) to S.C. and G.L., by FP7 CIG PCIG13-GA-2013-618697, Italian Ministry of Research FIRB RBAP11Z3YA_005, and by EFSD/Novo Nordisk Programme for Diabetes Research in Europe grant to L.S. F.G. and E.H-C. were supported by a DTIIMPORT FP7 Fellowship.

\section{AUTHOR CONTRIBUTIONS}

G.L., C.R., L.S., and S.C. conceived the project. F.C., F.G., A.Z., P.C., E.H.C., P.M, C.B, E.A., and R.F. performed experiments. F.C., P.L., G.G., C.R., P.B., L.S., S.C. and G.L. analysed results. F.C., S.C., L.S., P.B. and G.L. wrote the manuscript, which all Authors reviewed.

\section{DECLARATION OF INTERESTS}

The authors declare no competing interests. 


\section{REFERENCES}

Bean, C., Facchinello, N., Faulkner, G., and Lanfranchi, G. (2008). The effects of Ankrd2 alteration indicate its involvement in cell cycle regulation during muscle differentiation. Biochim Biophys Acta 1783, 1023-1035.

Biscontin, A., Casara, S., Cagnin, S., Tombolan, L., Rosolen, A., Lanfranchi, G., and De Pitta, C. (2010). New miRNA labeling method for bead-based quantification. BMC Mol Biol 11, 44.

Blanco, C.E., Sieck, G.C., and Edgerton, V.R. (1988). Quantitative histochemical determination of succinic dehydrogenase activity in skeletal muscle fibres. Histochem J 20, 230-243.

Boettger, T., Wust, S., Nolte, H., and Braun, T. (2014). The miR-206/133b cluster is dispensable for development, survival and regeneration of skeletal muscle. Skelet Muscle 4, 23.

Bostrom, P., Wu, J., Jedrychowski, M.P., Korde, A., Ye, L., Lo, J.C., Rasbach, K.A., Bostrom, E.A., Choi, J.H., Long, J.Z., et al. (2012). A PGC1-alpha-dependent myokine that drives brown-fat-like development of white fat and thermogenesis. Nature 481, 463-468.

Campbell, W.G., Gordon, S.E., Carlson, C.J., Pattison, J.S., Hamilton, M.T., and Booth, F.W. (2001). Differential global gene expression in red and white skeletal muscle. Am J Physiol Cell Physiol 280, C763-768.

Carrasco, M.A., and Hidalgo, C. (2006). Calcium microdomains and gene expression in neurons and skeletal muscle cells. Cell Calcium 40, 575-583.

Carrer, M., Liu, N., Grueter, C.E., Williams, A.H., Frisard, M.I., Hulver, M.W., Bassel-Duby, R., and Olson, E.N. (2012). Control of mitochondrial metabolism and systemic energy homeostasis by microRNAs 378 and 378*. Proc Natl Acad Sci U S A 109, 15330-15335.

Chemello, F., Bean, C., Cancellara, P., Laveder, P., Reggiani, C., and Lanfranchi, G. (2011). Microgenomic analysis in skeletal muscle: expression signatures of individual fast and slow myofibers. PLoS One 6, e16807.

Chemello, F., Mammucari, C., Gherardi, G., Rizzuto, R., Lanfranchi, G., and Cagnin, S. (2015). Gene expression changes of single skeletal muscle fibers in response to modulation of the mitochondrial calcium uniporter (MCU). Genom Data 5, 64-67.

Dumortier, O., Hinault, C., and Van Obberghen, E. (2013). MicroRNAs and metabolism crosstalk in energy homeostasis. Cell Metab 18, 312-324.

Friedlander, M.R., Chen, W., Adamidi, C., Maaskola, J., Einspanier, R., Knespel, S., and Rajewsky, N. (2008). Discovering microRNAs from deep sequencing data using miRDeep. Nat Biotechnol 26, 407-415.

Gan, Z., Rumsey, J., Hazen, B.C., Lai, L., Leone, T.C., Vega, R.B., Xie, H., Conley, K.E., Auwerx, J., Smith, S.R., et al. (2013). Nuclear receptor/microRNA circuitry links muscle fiber type to energy metabolism. J Clin Invest 123, 2564-2575.

Gomes, L.C., Di Benedetto, G., and Scorrano, L. (2011). During autophagy mitochondria elongate, are spared from degradation and sustain cell viability. Nat Cell Biol 13, 589-598.

Herrera, B.M., Lockstone, H.E., Taylor, J.M., Ria, M., Barrett, A., Collins, S., Kaisaki, P., Argoud, K., Fernandez, C., Travers, M.E., et al. (2010). Global microRNA expression profiles in insulin target tissues in a spontaneous rat model of type 2 diabetes. Diabetologia 53, 1099-1109. 
Hood, D.A., Irrcher, I., Ljubicic, V., and Joseph, A.M. (2006). Coordination of metabolic plasticity in skeletal muscle. J Exp Biol 209, 2265-2275.

$\mathrm{Hu}, \mathrm{W}$., and Coller, J. (2012). What comes first: translational repression or mRNA degradation? The deepening mystery of microRNA function. Cell Res 22, 1322-1324. Huang da, W., Sherman, B.T., and Lempicki, R.A. (2009). Systematic and integrative analysis of large gene lists using DAVID bioinformatics resources. Nat Protoc 4, 4457.

Jensen, J., Rustad, P.I., Kolnes, A.J., and Lai, Y.C. (2011). The role of skeletal muscle glycogen breakdown for regulation of insulin sensitivity by exercise. Front Physiol 2, 112.

Ji, J., Zhang, J., Huang, G., Qian, J., Wang, X., and Mei, S. (2009). Over-expressed microRNA-27a and 27b influence fat accumulation and cell proliferation during rat hepatic stellate cell activation. FEBS Lett 583, 759-766.

Kim, S.Y., Kim, A.Y., Lee, H.W., Son, Y.H., Lee, G.Y., Lee, J.W., Lee, Y.S., and Kim, J.B. (2010). miR-27a is a negative regulator of adipocyte differentiation via suppressing PPARgamma expression. Biochem Biophys Res Commun 392, 323-328.

Li, L.O., Grevengoed, T.J., Paul, D.S., Ilkayeva, O., Koves, T.R., Pascual, F., Newgard, C.B., Muoio, D.M., and Coleman, R.A. (2015). Compartmentalized acylCoA metabolism in skeletal muscle regulates systemic glucose homeostasis. Diabetes 64, 23-35.

Liesa, M., and Shirihai, O.S. (2013). Mitochondrial dynamics in the regulation of nutrient utilization and energy expenditure. Cell Metab 17, 491-506.

MacDonald, M.J., Tang, J., and Polonsky, K.S. (1996). Low mitochondrial glycerol phosphate dehydrogenase and pyruvate carboxylase in pancreatic islets of Zucker diabetic fatty rats. Diabetes 45, 1626-1630.

Mammucari, C., Gherardi, G., Zamparo, I., Raffaello, A., Boncompagni, S., Chemello, F., Cagnin, S., Braga, A., Zanin, S., Pallafacchina, G., et al. (2015). The mitochondrial calcium uniporter controls skeletal muscle trophism in vivo. Cell Rep 10, 1269-1279.

Martini, P., Sales, G., Brugiolo, M., Gandaglia, A., Naso, F., De Pitta, C., Spina, M., Gerosa, G., Chemello, F., Romualdi, C., et al. (2014). Tissue-specific expression and regulatory networks of pig microRNAome. PLoS One 9, e89755.

McCarthy, J.J. (2011). The MyomiR network in skeletal muscle plasticity. Exerc Sport Sci Rev 39, 150-154.

Mishra, P., Varuzhanyan, G., Pham, A.H., and Chan, D.C. (2015). Mitochondrial Dynamics is a Distinguishing Feature of Skeletal Muscle Fiber Types and Regulates Organellar Compartmentalization. Cell Metab 22, 1033-1044.

Murgia, M., Nagaraj, N., Deshmukh, A.S., Zeiler, M., Cancellara, P., Moretti, I., Reggiani, C., Schiaffino, S., and Mann, M. (2015). Single muscle fiber proteomics reveals unexpected mitochondrial specialization. EMBO Rep 16, 387-395.

Musa, C.V., Mancini, A., Alfieri, A., Labruna, G., Valerio, G., Franzese, A., Pasanisi, F., Licenziati, M.R., Sacchetti, L., and Buono, P. (2012). Four novel UCP3 gene variants associated with childhood obesity: effect on fatty acid oxidation and on prevention of triglyceride storage. Int J Obes (Lond) 36, 207-217.

Ortega, F.J., Mercader, J.M., Catalan, V., Moreno-Navarrete, J.M., Pueyo, N., Sabater, M., Gomez-Ambrosi, J., Anglada, R., Fernandez-Formoso, J.A., Ricart, W., et al. (2013). Targeting the circulating microRNA signature of obesity. Clin Chem 59, 781792.

Ortega, F.J., Mercader, J.M., Moreno-Navarrete, J.M., Rovira, O., Guerra, E., Esteve, E., Xifra, G., Martinez, C., Ricart, W., Rieusset, J., et al. (2014). Profiling of 
circulating microRNAs reveals common microRNAs linked to type 2 diabetes that change with insulin sensitization. Diabetes Care 37, 1375-1383.

Rambold, A.S., Kostelecky, B., Elia, N., and Lippincott-Schwartz, J. (2011). Tubular network formation protects mitochondria from autophagosomal degradation during nutrient starvation. Proc Natl Acad Sci U S A 108, 10190-10195.

Raz, V., Riaz, M., Tatum, Z., Kielbasa, S.M., and t Hoen, P.A.C. (2018). The distinct transcriptomes of slow and fast adult muscles are delineated by noncoding RNAs. FASEB J, fj201700861R.

Rottiers, V., and Naar, A.M. (2012). MicroRNAs in metabolism and metabolic disorders. Nat Rev Mol Cell Biol 13, 239-250.

Saeed, A.I., Sharov, V., White, J., Li, J., Liang, W., Bhagabati, N., Braisted, J., Klapa, M., Currier, T., Thiagarajan, M., et al. (2003). TM4: a free, open-source system for microarray data management and analysis. Biotechniques 34, 374-378.

Schiaffino, S., and Reggiani, C. (2011). Fiber types in mammalian skeletal muscles. Physiol Rev 91, 1447-1531.

Schrepfer, E., and Scorrano, L. (2016). Mitofusins, from Mitochondria to Metabolism. Mol Cell 61, 683-694.

Sears, B., and Perry, M. (2015). The role of fatty acids in insulin resistance. Lipids Health Dis $14,121$.

Shannon, P., Markiel, A., Ozier, O., Baliga, N.S., Wang, J.T., Ramage, D., Amin, N., Schwikowski, B., and Ideker, T. (2003). Cytoscape: a software environment for integrated models of biomolecular interaction networks. Genome Res 13, 2498-2504.

Sharan, R., Maron-Katz, A., and Shamir, R. (2003). CLICK and EXPANDER: a system for clustering and visualizing gene expression data. Bioinformatics 19, 17871799.

Shirasaki, T., Honda, M., Shimakami, T., Horii, R., Yamashita, T., Sakai, Y., Sakai, A., Okada, H., Watanabe, R., Murakami, S., et al. (2013). MicroRNA-27a regulates lipid metabolism and inhibits hepatitis $\mathrm{C}$ virus replication in human hepatoma cells. $\mathrm{J}$ Virol 87, 5270-5286.

Tibshirani, R., Hastie, T., Narasimhan, B., and Chu, G. (2002). Diagnosis of multiple cancer types by shrunken centroids of gene expression. Proc Natl Acad Sci U S A 99, 6567-6572.

Tusher, V.G., Tibshirani, R., and Chu, G. (2001). Significance analysis of microarrays applied to the ionizing radiation response. Proc Natl Acad Sci U S A 98, 5116-5121.

van Rooij, E., Quiat, D., Johnson, B.A., Sutherland, L.B., Qi, X., Richardson, J.A., Kelm, R.J., Jr., and Olson, E.N. (2009). A family of microRNAs encoded by myosin genes governs myosin expression and muscle performance. Dev Cell 17, 662-673.

Vaughan, R.A., Gannon, N.P., Barberena, M.A., Garcia-Smith, R., Bisoffi, M., Mermier, C.M., Conn, C.A., and Trujillo, K.A. (2014). Characterization of the metabolic effects of irisin on skeletal muscle in vitro. Diabetes Obes Metab 16, 711718.

Vowinckel, J., Hartl, J., Butler, R., and Ralser, M. (2015). MitoLoc: A method for the simultaneous quantification of mitochondrial network morphology and membrane potential in single cells. Mitochondrion 24, 77-86.

Wang, T., Li, M., Guan, J., Li, P., Wang, H., Guo, Y., Shuai, S., and Li, X. (2011). MicroRNAs miR-27a and miR-143 regulate porcine adipocyte lipid metabolism. Int J Mol Sci 12, 7950-7959.

Wu, H., Gallardo, T., Olson, E.N., Williams, R.S., and Shohet, R.V. (2003). Transcriptional analysis of mouse skeletal myofiber diversity and adaptation to endurance exercise. J Muscle Res Cell Motil 24, 587-592. 
Xiong, X.Q., Chen, D., Sun, H.J., Ding, L., Wang, J.J., Chen, Q., Li, Y.H., Zhou, Y.B., Han, Y., Zhang, F., et al. (2015). FNDC5 overexpression and irisin ameliorate glucose/lipid metabolic derangements and enhance lipolysis in obesity. Biochim Biophys Acta 1852, 1867-1875. 


\section{FIGURE LEGENDS}

\section{Figure 1: Transcriptional signatures of myofibers from soleus and EDL mouse} muscles.

(A) Single isolated myofibers were dissociated from soleus and EDL mouse muscles and classified by MyHC isoform pattern. (B) Cluster analysis of the DE microarray probes identifies two main signatures (1 and 2) that reflect fiber metabolism (oxidative and glycolytic). Oxidative signature (1) is further divided in two groups (1a and $1 \mathrm{~b}$ ) that we named transcriptional slow (tS) and intermediate (tI). MyHC isoform composition was indicated for pure (MyHC) or hybrid (Hyb) types. Myofibers of the same type were numbered from 1 to 10 and marked with the muscle of origin ( $\mathrm{S}$ for soleus, and E for EDL) and the mouse number (parentheses). The heat-map shows the expression of DE genes involved in metabolism as identified by GO analysis (Table 1) (Blue is for low expression, red for high and grey for under detection limit). On the left are indicated gene functions: FA metabolic process (FA), acetyl-CoA metabolic process (AcoA), electron transport chain (ETC) and glucose metabolism (Gluc). (C) miRNA libraries were constructed and amplified using the SMART technology and profiled by NGS. A total of 137 miRNAs expressed in at least one myofiber transcriptional type was identified: 81 miRNAs were found more expressed in tS myofibers, 84 in tI myofibers, and 75 in $\mathrm{tF}$ myofibers. 33 miRNAs (including the myomiRs miR-1a-3p and -133a-3p) are generally expressed across all myofibers. (D) Networks of post-transcriptional regulation in $\mathrm{tS}$, $\mathrm{tI}$ and $\mathrm{tF}$ myofibers. Triangles are for miRNAs and squares for mRNAs, while colors reflect their expression levels). GO enrichment analysis was performed on genes of the two sub-networks, and only terms 
with an enrichment score $>1.3$ are listed (the highest at the top). For larger images see Figure S5.

Figure 2: Effects of miR-27a-3p and -142-3p on mitochondrial morphology and energy metabolism: in vitro studies.

(A) Relative quantification (RQ) by qRT-PCR of the selected miRNAs. miRNA expression levels were tested in a slow muscle (soleus, SOL) and in a fast muscle (EDL, used for data normalization). Each experiment was performed at least in triplicate. (B) NGS results for the selected miRNAs on $\mathrm{tS}$, tI and $\mathrm{tF}$ myofibers. Expression levels were normalized on tS myofibers. (C) Quantification of the fragmentation index $f$ of mitochondrial networks after miRNA transfections. $n=$ at least 20 for each experiment. (D) Representative fluorescence microscopy images of single cells with different levels of mitochondria fragmentation. Mitochondria were stained with anti-TOM20 and imaged by confocal microscopy. Images of each cell were captured at different focal depths and then processed by focal plane merging and convolution. Scale bar is $10 \mu \mathrm{m}$. (E) and (G) Bioenergetic profiles of transfected myoblasts in glucose or palmitate substrate medium. OCR of $\mathrm{C} 2 \mathrm{C} 12$ myoblasts transfected with miRNA mimics was evaluated with the sensitive Seahorse technology after 72 hours of transfection. Bioenergetic profiles of cells were measured in a basal state and after the addition of oligomycin $(\mathrm{O}$, to inhibit ATP synthase), FCCP (F, to uncouple ATP synthesis from the electron transport chain), and rotenone and antimycin A ( R and AA, to block respectively complexes I and III of the electron transport chain). In palmitate medium, lipid specific respiration was analysed by acute Cpt1 inhibition by etomoxir. miRNA transfections were independently replicates at least three times, and for each one five technical replicates 
were measured. $(\mathrm{F})$ and $(\mathrm{H})$ Parameters of mitochondrial function of transfected myoblasts in glucose or palmitate resulting from the bioenergetic profiles. (I) Quantification of the fragmentation index $f$ of mitochondrial networks after miR-27a$3 p$ or miR-142-3p transfections in palmitate medium. Control experiments performed with Bovine Serum Albumine alone (BSA, necessary for conjugate palmitate), confirmed that miR-142-3p mitochondrial fragmentation is induced by palmitate (data not shown). For each experiment, $n=$ at least 20. (J) C2C12 myoblasts were transfected with a scramble sequence as control, with miR-27a-3p, or with miR-1423p. Cells were lysed and glycogen content was quantified using a luminometer. Data are the mean of two replicates for three independent transfection experiments $(n=6)$. (K) Transfected myoblasts were stained for lipids with BODIPY fluorophore and fluorescence positive cells were counted by flow-cytometry. Data are the mean of two replicates for three independent transfection experiments $(n=6)$. (L) Lipids were extracted from myoblasts transfected with a control scramble sequence or with miR142-3p, and measured with liquid chromatography-mass spectrometry (LC-MS) technology. Classes of lipids significantly different between samples are represented in the figure (Lyso-PC: lysophosphatidylcholine, PC: phosphatidylcholine, PI: phosphatidylinositol, DAG: diacylglycerol, TAG: triacylglycerol). Lipid quantification was measured as arbitrary units (A.U.) of signal areas of chromatograms and normalized to the number of cells relative to each sample. Number of independent experiments $(n)$ per condition $=4$. In the entire figure: Scr is the control transfected with a scrambled sequence; error bars are SEM; adjusted Pvalues of ANOVA statistical test were indicated as follow: $*<0.05, * *<0.01, * * *<$ 0.001, and they are referred in comparison to the control. 
Figure 3: Identification and characterization of transcripts that are altered by miR-27a-3p and miR-142-3p over-expression.

(A) Expression profiling of myoblasts transfected with miR-27a-3p or miR-142-3p showed that most targets identified in myofiber mRNAs-miRNAs interaction networks of Figure 2C are down-regulated. 32 transcripts out of 48 targets of miR$27 \mathrm{a}-3 \mathrm{p}(66.7 \%)$ are down-regulated in $\mathrm{C} 2 \mathrm{C} 12$ myoblasts upon the over-expression of that miRNA. Similarly, the over-expression of miR-142-3p resulted in the downregulation of 17 out of 23 targets (73.9\%). Tables flanking pie graphs list the expression values ( $\log _{2}$ fold change "FC") of down-regulated miRNA targets in transfected myoblasts in comparison with myoblasts transfected with a scrambled sequence as control (Scr). (B) Luciferase assays were performed to demonstrate the direct interaction between the two miRNAs and their targets. Part of the 3'-UTR sequence containing the miRNA putative interaction site (or not containing, ctr) was cloned in pmirGLO vector. Firefly luciferase (reporter gene) and renilla luciferase (control reporter for normalization) activities were measured after the transfection in C2C12 cells together with miRNA mimics or miRNA Scr sequence. Data are expressed as mean of at least five replicates; error bars are SEM; P-values of t-test are indicated as follow: $*<0.05, * * *<0.001$. (C) Relative quantification (RQ) by qRTPCR of specific transcripts, codifying for relevant metabolic enzymes, in mouse EDL muscles transfected with miR-27a-3p or $-142-3 p$ mimics. These in vivo experiments confirm that a) miR-27a-3p up-regulation inhibits the expression of Pgm2 and Gaa genes, and b) miR-142-3p up-regulation diminishes the expression of Fndc5 and Cpt2. Moreover, when miR-142-3p is up-regulated in muscles, Plin2, a marker of lipid droplets, is over-expressed and the lipase hormone sensitive (Lipe) is under- 
expressed. Data are expressed as mean of three independent transfections; error bars are SEM; P-values of t-test are indicated as follow: $*<0.05, * * *<0.001$.

Figure 4: Alterations of fuel availability induced by miR-27a-3p and miR-1423p: in vivo studies.

(A) Representative cross sections of mouse tibialis anterior stained with PAS to detect glycogen, after one week of transfection with a scramble sequence as control (Scr), with miR-27a-3p, or with miR-142-3p. Scale bar is $200 \mu \mathrm{m}$. (B) The intensities of PAS color pixel were graphed in Tukey box-plots. Number of independent experiments $(n)$ per condition $=3$. (C) Electron microscopy of longitudinal $($ LONG) and transverse (TRANS) sections of transfected gastrocnemius muscle. Arrows indicate glycogen granules in muscles over-expressing miR-27a-3p, or lipid droplets in muscles over-expressing miR-142-3p. Scale bar is $500 \mathrm{~nm}$. (D) Representative cross sections of mouse tibialis anterior stained for lipids with BODIPY, after one week of transfection with a scramble sequence as control, with miR-27a-3p, or with miR-142-3p. Scale bar is $50 \mu \mathrm{m}$. (E) BODIPY fluorescence pixel intensities were graphed in Tukey box-plots. Number of independent experiments $(n)$ per condition $=$

3. (F) Representative cross section of mouse muscle gastrocnemius after one week of transfection. Oxidative myofibers are positive to succinate dehydrogenase (SDH) staining (blue color). Scale bar is $100 \mu \mathrm{m}$. (G) Quantification of SDH-positive fibers. At least 2,500 fibers were analyzed for each transfection. Error bars are SEM; adjusted P-values of ANOVA statistical test is indicated as follows: $*<0.05$, and referred in comparison to the control. 


\section{Figure 5: Role of miR-27a-3p and -142-3p in myofiber metabolism.}

(A) A model that integrates the circuits by which miR-27a-3p and $-142-3 p$ may orchestrate glycogen and FA metabolism in tI myofibers. (B) A more-detailed scheme representing miRNA modulation of myofiber metabolism. For all the components of the scheme, blue color indicates low levels and red color high levels. Asterisks indicate the miRNAs of interest. 


\section{TABLES}

Table 1: Gene Ontology analysis of genes differentially expressed among transcriptional myofiber types.

\begin{tabular}{|c|c|c|}
\hline & $\begin{array}{l}\text { Enrichment } \\
\text { Score }\end{array}$ & P-value \\
\hline \multicolumn{3}{|c|}{$\begin{array}{l}\text { Probes overexpressed in tS myofibers (cluster 1, } 114 \text { probes, } 94 \\
\text { DAVID IDs) }\end{array}$} \\
\hline Muscle tissue development & 2.14 & $3.03 \mathrm{E}-04$ \\
\hline Cytoskeletal protein binding & 1.96 & $4.57 \mathrm{E}-04$ \\
\hline \multicolumn{3}{|c|}{$\begin{array}{l}\text { Probes overexpressed in tS and tI myofibers (clusters } 2 \text { and 3, } 806 \\
\text { probes, } 643 \text { DAVID IDs) }\end{array}$} \\
\hline Mitochondrion & 23.80 & $9.37 \mathrm{E}-33$ \\
\hline Fatty acid metabolic process & 8.51 & $6.12 \mathrm{E}-12$ \\
\hline Myofibril & 8.18 & $6.95 \mathrm{E}-10$ \\
\hline Electron transport chain & 4.65 & $5.71 \mathrm{E}-06$ \\
\hline Acetyl-CoA metabolic process & 3.52 & $1.87 \mathrm{E}-07$ \\
\hline \multicolumn{3}{|c|}{$\begin{array}{l}\text { Probes overexpressed in tI myofibers (cluster 4, } 95 \text { probes, } 85 \\
\text { DAVID IDs) }\end{array}$} \\
\hline Mitochondrion & 7.95 & $2.40 \mathrm{E}-18$ \\
\hline Electron transport chain & 5.63 & $4.12 \mathrm{E}-13$ \\
\hline \multicolumn{3}{|c|}{$\begin{array}{l}\text { Probes overexpressed in tI and tF myofibers (cluster 5, } 240 \text { probes, } \\
123 \text { DAVID IDs) }\end{array}$} \\
\hline Glucose metabolic process & 9.23 & $6.64 \mathrm{E}-10$ \\
\hline Muscle contraction & 2.40 & $2.57 \mathrm{E}-03$ \\
\hline Myofibril & 1.98 & $8.80 \mathrm{E}-04$ \\
\hline \multicolumn{3}{|c|}{$\begin{array}{l}\text { Probes overexpressed in tF myofibers (clusters } 6 \text { and } 7,638 \text { probes, } \\
506 \text { DAVID IDs) }\end{array}$} \\
\hline Sarcoplasmic reticulum & 8.06 & $8.06 \mathrm{E}-11$ \\
\hline Myofibril & 5.61 & $6.27 \mathrm{E}-07$ \\
\hline Glutathione transferase activity & 3.16 & $2.13 \mathrm{E}-03$ \\
\hline Glucose metabolic process & 2.90 & $1.59 \mathrm{E}-05$ \\
\hline Muscle contraction & 2.79 & $3.73 \mathrm{E}-04$ \\
\hline
\end{tabular}

Gene Ontology (GO) enrichment analysis of the 1,936 DE microarray probes was separately performed on genes that share common expression patterns among the 3 transcriptional myofiber signatures (tS, tI, and tF) using DAVID bioinformatic tool. One representative GO term from each functional annotation cluster with an 
enrichment score cutoff $>1.3$ (the higher, the better) and P-values $<0.05$ (the lower, the better) was listed. Overlapping functional terms were ignored and only groups from the top 5 clusters are shown (additional information in Dataset S5). 
Table S1: Results of myofiber typing.

\begin{tabular}{|c|c|c|c|c|c|c|c|c|}
\hline & 1 & $2 A$ & $2 \mathrm{~A} / 2 \mathrm{X}$ & $2 X$ & $2 \mathrm{X} / 2 \mathrm{~B}$ & $2 B$ & Others* & Total \\
\hline Soleus & 46 & 11 & 15 & 21 & 1 & 1 & $\begin{array}{c}5 \\
\begin{array}{c}(1 / 2 \mathrm{~A}: 2,1 / 2 \mathrm{X}: 1, \\
2 \mathrm{X} / 2 \mathrm{~B}: 1.2 \mathrm{~B} 1,\end{array}\end{array}$ & 100 \\
\hline EDL & 0 & 1 & 0 & 2 & 29 & 64 & $\begin{array}{c}4 \\
(1 / 2 \mathrm{~B}: 1,2 \mathrm{~A}: 1, \\
2 \mathrm{~A} / 2 \mathrm{~B}: 1, \\
2 \mathrm{~A} / 2 \mathrm{X} / 2 \mathrm{~B}: 1)\end{array}$ & 100 \\
\hline
\end{tabular}

Single fibers were enzymatically dissociated from soleus $(n=13)$ and EDL $(n=9)$ mouse muscles. A small fragment of each isolated myofiber was characterized on the basis of MyHC isoform protein expression determined by SDS-PAGE. The distribution of fiber types mirrors the properties of the two muscles analyzed. All major adult fiber types were identified together with the most frequent hybrid combinations. * These myofibers, due to not typical MyHC composition, were not processed for expression profiling. 
Table S2: miRNAs most expressed in skeletal muscle fibers.

\begin{tabular}{|c|c|c|c|}
\hline miRNA & $\begin{array}{c}\text { Common } \\
\text { name }\end{array}$ & $\begin{array}{c}\text { Expression } \\
\text { value }\end{array}$ & Representative literature \\
\hline $\begin{array}{l}\text { mmu-miR-1a- } \\
3 p\end{array}$ & $\operatorname{miR}-1$ & $>14$ & \multirow{3}{*}{$\begin{array}{l}\text { - The role of microRNA-1 and microRNA-133 in skeletal } \\
\text { muscle proliferation and differentiation (Chen JF et al., } \\
\text { 2006). } \\
\text { Myogenic factors that regulate expression of muscle- } \\
\text { specific microRNAs (Rao PK et al., 2006). } \\
\text { microRNA-1 and microRNA-206 regulate skeletal } \\
\text { muscle satellite cell proliferation and differentiation by } \\
\text { repressing Pax7 (Chen JF et al., 2010). } \\
\text { Transgenic overexpression of miR-133a in skeletal } \\
\text { muscle (Deng Z et al., 2011). } \\
\text { miR-133b, a muscle-specific microRNA, is a novel } \\
\text { prognostic marker that participates in the progression of } \\
\text { human colorectal cancer via regulation of CXCR4 } \\
\text { expression (Duan FT et al., 2013). } \\
\text { MicroRNA-133 controls brown adipose determination in } \\
\text { skeletal muscle satellite cells by targeting Prdm16 (Yin } \\
\text { H et al., 2013). }\end{array}$} \\
\hline $\begin{array}{l}\text { mmu-miR- } \\
133 a-3 p\end{array}$ & $\begin{array}{l}\mathrm{miR}- \\
133 \mathrm{a}\end{array}$ & $>14$ & \\
\hline $\begin{array}{l}\text { mmu-miR- } \\
133 b-5 p\end{array}$ & $\begin{array}{l}\text { miR- } \\
133 b^{*}\end{array}$ & $>13$ & \\
\hline $\begin{array}{l}\text { mmu-miR-29a- } \\
3 p\end{array}$ & miR-29a & $>13$ & \multirow{2}{*}{ 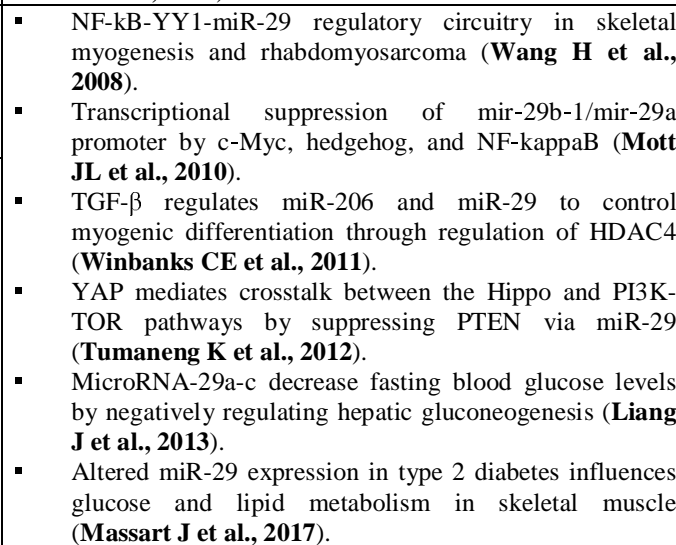 } \\
\hline $\begin{array}{l}\text { mmu-miR-29c- } \\
3 p\end{array}$ & $\operatorname{miR}-29 c$ & $>14$ & \\
\hline mmu-let-7a-5p & let-7a & $>13$ & \multirow{6}{*}{ 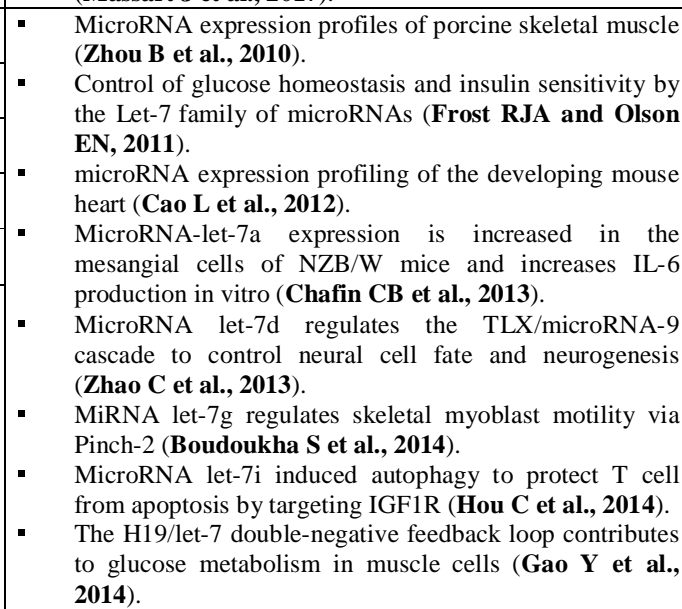 } \\
\hline mmu-let-7c-5p & let-7c & $>13$ & \\
\hline mmu-let-7d-5p & let-7d & $>13$ & \\
\hline mmu-let-7f-5p & let-7f & $>14$ & \\
\hline mmu-let-7g-5p & let-7g & $>13$ & \\
\hline mmu-let-7i-5p & let-7i & $>13$ & \\
\hline $\begin{array}{l}\text { mmu-miR-191- } \\
5 \mathrm{p}\end{array}$ & miR-191 & $>13$ & $\begin{array}{l}\text { Myogenic factors that regulate expression of muscle- } \\
\text { specific microRNAs (Rao PK et al., 2006). } \\
\text { miR-191 regulates mouse erythroblast enucleation by } \\
\text { down-regulating Riok3 and Mxi1 (Zhang L et al., 2011). } \\
\text { PU.1 promotes miR-191 to inhibit adipogenesis in 3T3- } \\
\text { L1 preadipocytes (Ji S et al., 2014). } \\
\text { miR-191 suppresses angiogenesis by activation of NF-kB } \\
\text { signaling (Gu Y et al., 2017). }\end{array}$ \\
\hline $\begin{array}{l}\text { mmu-miR-378- } \\
3 p\end{array}$ & $\operatorname{miR}-378$ & $>13$ & $\begin{array}{l}\text { MicroRNA-378 targets the myogenic repressor MyoR } \\
\text { during myoblast differentiation (Gagan J at al., 2011). } \\
\text { - Control of mitochondrial metabolism and systemic }\end{array}$ \\
\hline
\end{tabular}




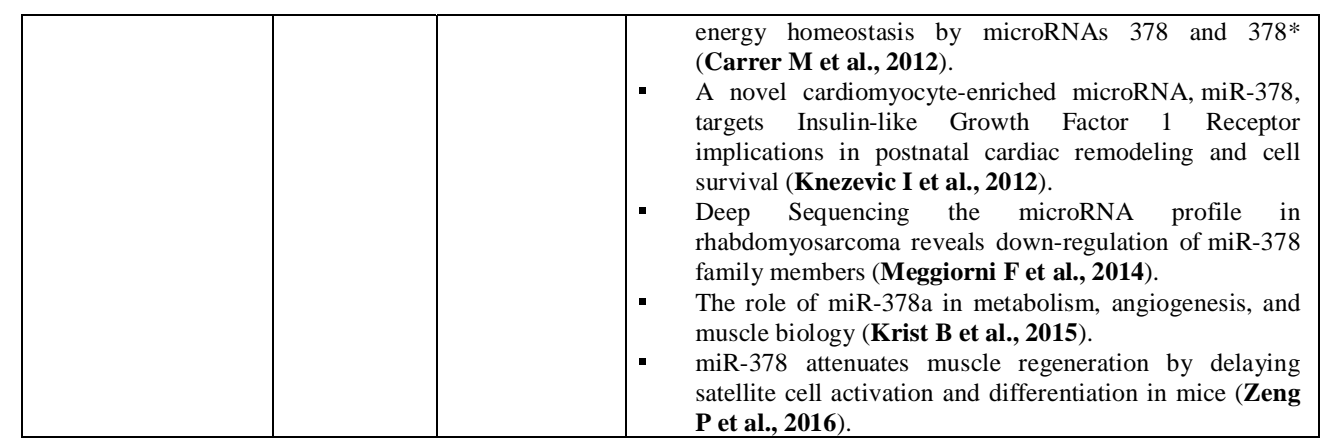

NGS experiments performed on single isolated myofibers identified a list of 13 miRNAs highly expressed. Expression values are $\log _{2}$ of sequencing results normalized as counts of reads per million $(+1)$ and refer to all the three transcriptional signatures. For each miRNA or miRNA family, a list of representative literature is cited in order to elucidate their role in skeletal muscle biology. 
Table S3: Expression changes of genes involved in the synthesis of metabolic enzymes.

\begin{tabular}{|c|c|c|c|c|}
\hline \multicolumn{5}{|c|}{ Myoblasts transfected with miR-27a mimic } \\
\hline & Gene & Probe & Description & Fold change \\
\hline \multirow{3}{*}{$\underline{\text { Glycogenolysis }}$} & Pygm & A_55_P1961913 & Muscle glycogen phosphorylase & -0.80 \\
\hline & Pgm2 & A_55_P1987483 & Phosphoglucomutase 2 & -0.50 \\
\hline & Gaa & A_55_P2024431 & Glucosidase, alpha, acid & -0.58 \\
\hline \multicolumn{5}{|c|}{ Myoblasts transfected with miR-142 mimic } \\
\hline & Gene & Probe & Description & Fold change \\
\hline \multirow{9}{*}{$\begin{array}{l}\text { Fatty acid } \\
\underline{\text { metabolism }}\end{array}$} & Acsl1 & A_51_P496432 & $\begin{array}{l}\text { Acyl-CoA synthetase long- } \\
\text { chain family member } 1\end{array}$ & 0.41 \\
\hline & Fndc5 & A_52_P213696 & $\begin{array}{l}\text { Fibronectin type III domain } \\
\text { containing } 5\end{array}$ & -4.14 \\
\hline & Acsl3 & A_55_P2165324 & $\begin{array}{l}\text { Acyl-CoA synthetase long- } \\
\text { chain family member } 3\end{array}$ & -0.64 \\
\hline & Acsl6 & A_51_P380699 & $\begin{array}{l}\text { Acyl-CoA synthetase long- } \\
\text { chain family member } 6\end{array}$ & -2.87 \\
\hline & Cpt2 & A_55_P2074174 & Carnitine palmitoyltransferase 2 & -0.85 \\
\hline & Acad10 & A_55_P2088490 & $\begin{array}{l}\text { Acyl-CoA dehydrogenase } \\
\text { family, member } 10\end{array}$ & -0.47 \\
\hline & Acad12 & A_52_P671700 & $\begin{array}{l}\text { Acyl-CoA dehydrogenase } \\
\text { family, member } 12\end{array}$ & -2.09 \\
\hline & Ucp2 & A_51_P297105 & $\begin{array}{l}\text { Uncoupling protein } 2 \\
\text { (mitochondrial, proton carrier) }\end{array}$ & -3.84 \\
\hline & Ucp3 & A_66_P108247 & $\begin{array}{l}\text { Uncoupling protein } 3 \\
\text { (mitochondrial, proton carrier) }\end{array}$ & -4.18 \\
\hline \multirow{3}{*}{$\underline{\text { Lipid droplets }}$} & Plin1 & A_51_P244497 & Perilipin 1 & 1.47 \\
\hline & Plin2 & A_55_P1999648 & Perilipin 2 & 0.48 \\
\hline & Plin4 & A_51_P495780 & Perilipin 4 & 1.53 \\
\hline
\end{tabular}

Expression levels of selected differentially expressed genes coding for protein involved in glycogenolysis, FA metabolism or markers of lipid droplets in myoblasts transfected with miR-27a-3p or miR-142-3p mimics. $\log _{2}$ fold change is in comparison with myoblasts transfected with a scrambled sequence. Blue colour indicates under-expression, red colour over-expression. 
Table S4: Primers used for qRT-PCRs and luciferase assays.

\begin{tabular}{|l|l|l|}
\hline Gene Symbol & Primer LEFT & Primer RIGHT \\
\hline Actn3 & GACGACTTCCGAGCTTGTCT & CTGCCAGGATTTTGAAGGAG \\
\hline Cpt2 & CCAAAGAAGCAGCGATGG & TAGAGCTCAGGCAGGGTGA \\
\hline Fndc5 & TTGTGGTCCTCTTCATGTGG & TTATTGGGCTCGTTGTCCTT \\
\hline Gaa & CCTTTGGAGTGATCGTTCGT & CCACGGTTGTGTTCAGCA \\
\hline Lipe & CTTCCTGCAAGAGTATGTCACG & ATGGCAGGTGTGAACTGGA \\
\hline Myh2 & TTGGTGGATAAACTCCAGGC & CAGCTTGTTGACCTGGGACT \\
\hline Myh4 & TAGGGTGAGGGAGCTTGAAA & GTTTGTCCACCAAGTCCTGC \\
\hline Myh7 & AGCAGGAGCTGATTGAGACC & TGTGATAGCCTTCTTGGCCT \\
\hline Myl3 & ACGCTGGGTGAGAGACTGAC & GAGTCCGAACCACTCCTTCC \\
\hline Pgm2 & GGCCGCTTCTACATGACC & TAACCAGGCGACCAATCC \\
\hline Plin2 & CTCCACTCCACTGTCCACCT & GCTTATCCTGAGCACCCTGA \\
\hline Tpm3 & CTGGAGGAGGAGCTGAAGAA & TCAGTTTCTGGGCATAGAGC \\
\hline Txn1 & TCCAATGTGGTGTTCCTTGA & GGCTTCAAGCTTTTCCTTGTT \\
\hline Fndc5_luc & $\begin{array}{l}\text { CTCGGAGCTCGCTCTGGGAGAA } \\
\text { AGCAGCTA }\end{array}$ & $\begin{array}{l}\text { CTCGTCTAGAAGTAAGGGGC } \\
\text { AGAGGAGAGG }\end{array}$ \\
\hline Fndc5_luc_ctr & $\begin{array}{l}\text { CTCGGAGCTCGGAAAGCCGTTT } \\
\text { ACACCAGA }\end{array}$ & $\begin{array}{l}\text { CTCGTCTAGAGTTCAGCAGG } \\
\text { GATGGAAGTC }\end{array}$ \\
\hline Gaa_luc & $\begin{array}{l}\text { CTCGGAGCTCCTGCAGAGACAG } \\
\text { AGCTGGTG }\end{array}$ & $\begin{array}{l}\text { CTCGTCTAGATGCTTCCAAA } \\
\text { CAATATCCTTTTAT }\end{array}$ \\
\hline Gaa_luc_ctr & $\begin{array}{l}\text { CTCGGAGCTCCATCCCTGTCTC } \\
\text { ACTCGTCTAGATGGAGGCCTC } \\
\text { TGTAAACGAC }\end{array}$ \\
\hline Pgm2_luc & $\begin{array}{l}\text { CTCGGAGCTCTGCACCTGTGTC } \\
\text { TGTTCCTT }\end{array}$ & $\begin{array}{l}\text { CTCGTAGAGCAGATGGGA } \\
\text { GATCAAAAT }\end{array}$ \\
\hline Pgm2_luc_ctr & $\begin{array}{l}\text { TTCGGAGCTCCCCTGATCTCCA } \\
\text { AAATGGCTGT }\end{array}$ \\
\hline
\end{tabular}

Primers were designed using Primer3Plus. 


\section{MATERIALS AND METHODS}

\section{$\underline{\text { Ethic statement }}$}

All aspects of animal care and experimentation were performed in accordance with the Guide for the Care and Use of Laboratory Animals published by the National Institutes of Health (NIH Publication No. 85-23, Revised 1996) and the Italian regulations (DL 116/92) concerning the maintenance and use of laboratory animals. Experimental procedures were approved by the Ethical Committee of the University of Padova.

\section{$\underline{\text { Animals }}$}

Hindlimb muscles were collected from wild-type CD1 male mice at 3 months of age (Charles River, weight: 33 - 35 g). Mice were housed in a normal environment, provided with food and water and were killed by rapid cervical dislocation to minimize suffering.

\section{Screening of soleus and EDL myofibers}

Enzymatic dissociation and classification of myofibers from soleus and EDL mouse muscles were fully described in our previous work (Chemello et al., 2011). Briefly, after collagenase treatment, each single isolated myofiber was cut and about one-third of the fiber was used for MyHC protein isoform classification by SDS-PAGE whereas the remaining part was immediately immersed in TRIzol Reagent (Thermo Fisher Scientific) for RNA purification. All myofibers were collected within $45 \mathrm{~min}$. from muscle disaggregation. 


\section{$\underline{\text { RNA isolation }}$}

Single isolated myofiber were separately lysed in $250 \mu$ l of TRIzol Reagent (Thermo Fisher Scientific) and RNA was extracted in the aqueous phase following the manufacturer's instructions. $70 \%$ ethanol was added before column purification. For microarray experiments, total RNA was purified using the RNeasy Micro Kit (Qiagen), whereas, for miRNA analyses, separation of small and large RNAs was performed combining it with the miRNeasy Mini Kit (Qiagen) as suggested by manufacturer's protocol. RNAs from whole muscles or cells were isolated using the standard phenol-chloroform extraction with TRIzol Reagent (Thermo Fisher Scientific) followed by isopropanol precipitation.

\section{Microarray profiles}

\section{Amplification and labeling of RNA from single fibers}

In order to obtain a sufficient amount of cDNA for microarray experiments, RNA purified from a single myofiber was exponentially amplified using the TransPlex Whole Transcriptome Amplification 2 Kit (Sigma-Aldrich) in accordance with the manufacturer's instructions. RNA was reverse-transcribed in a cDNA library, and library was amplified for 18 cycles, below the amplification "plateau" observed in a PCR test reaction. To remove the residual primers and nucleotides, amplification product was purified with the GenElute PCR Clean-up columns (Sigma-Aldrich). Resulting cDNA was quantified with Nanodrop ND-1000 spectrophotometer (Thermo Fisher Scientific). $2 \mu \mathrm{g}$ of amplified-purified cDNA were directly labeled using the Genomic DNA Enzymatic Labeling Kit (Agilent Technologies) as described by the proprietary protocol. The kit uses random primers and the exo-Klenow fragment to 
directly label cDNA samples with Cy3-dUTP nucleotides. Labeled cDNA was purified using the Amicon 30kDa filters (Millipore) and quantified using NanoDrop ND-1000 spectrophotometer (Thermo Fisher Scientific). On average, cDNA yield was about $4 \mu \mathrm{g}$ and the specific activity was of 30 pmol Cy3 per $\mu \mathrm{g}$ cDNA.

\section{Amplification and labeling of RNA from cells}

RNAs obtained from cells were amplified and labeled starting from $150 \mathrm{ng}$ and using the Low Input Quick Amp Labeling Kit, one-color (Agilent Technologies) as described by the proprietary protocol. Labeled cRNA was purified with miRNeasy Mini Kit (Qiagen) and quantified using NanoDrop ND-1000 spectrophotometer (Thermo Fisher Scientific). On average, cRNA yield was about $10 \mu \mathrm{g}$ and the specific activity was of 35 pmol Cy3 per $\mu$ g cRNA.

\section{Hybridization}

Microarray experiments were performed with SurePrint G3 Mouse Gene Expression 8x60K microarray platforms (Agilent Technologies) containing 8 arrays per slide and consisting of 39,430 60mer oligonucleotide probes for Entrez Gene RNAs (GPL10787). $800 \mathrm{ng}$ of labeled sample target were mixed with $5 \mu$ l of 10X Blocking Agent (Agilent Technologies) and water to a final volume of $25 \mu$ l. Samples were denatured at $95^{\circ} \mathrm{C}$ for 2 min. and added to $25 \mu$ of $2 \mathrm{X}$ GEx Hybridization Buffer HIRPM (Agilent Technologies). $40 \mu \mathrm{lmix}$ was dispensed onto the array. Slides were loaded into the Agilent SureHyb chambers and hybridization was performed in a hybridization oven at $65^{\circ} \mathrm{C}$ for 17 hours with $10 \mathrm{rpm}$ rotation. Finally, slides were washed using Wash Buffer Kit (Agilent Technologies) and dried at room temperature. 


\section{$\underline{\text { Microarray data analysis }}$}

\section{Data pre-processing}

Microarray slides were scanned using G2505C scanner (Agilent Technologies) at 3 $\mu \mathrm{m}$ resolution. Probes features were extracted using the Feature Extraction Software v. 10.7.3.1 with GE_1_Sep09 protocol (Agilent Technologies). Intra-array normalizations were directly performed by the Feature Extraction Software. The raw data are available in the GEO database (GSE98328). Quantile inter-arrays normalization was performed using the Expander software (Sharan et al., 2003). Data were $\log _{2}$ transformed and not available (NA) value was assigned if Found and/or Well Above Background flags were not positive. Expression values of probes with the same sequence were mediated. In myofiber microarray experiments, 10 myofibers per each of 6 myofiber types (classified by MyHC protein expression, total: 60 microarrays) were analyzed. Only probes with at least 8 of 10 available values in at least one myofiber type were taken into consideration for the further analyses. In cells microarray experiments, 6 independent replicates for miRNA mimic transfected cells, and 4 independent replicates for cells transfected with a scrambled sequence (used as control) were analyzed. Only probes with at least $75 \%$ of available values in at least one condition were taken into consideration for the further analyses.

\section{Statistical analyses of microarray data}

Microarray data were analyzed using the MultiExperiment Viewer (MeV, Ver. 4.8), a tool of TM4 Microarray Software Suite (Saeed et al., 2003). To identify the DE probes among myofibers, we performed one-way analysis of variance (ANOVA). Each sample was assigned at one of the 6 groups identified by the MyHC protein 
classification. Probes with an adjusted Bonferroni P-value (based on 1,000 permutations) lower than 0.01 were considered as DE. In addition, to improve the number of genes differentially expressed, probes not recognized by the statistical test and exclusively expressed in 1 or 2 clusters were manually added to the list. Samples (myofibers) were hierarchically clustered using the Pearson's correlation distance and average linkage method. DE probes were grouped in 8 different clusters by K-means clustering, on the basis of their expression. Intensity values of the probes of the same cluster and of the same transcriptional class were mediate and differences among classes were considered statistically and biologically significant if the adjusted Pvalue generated by ANOVA was < 0.01 (calculated with GraphPad Prism software) and the difference of means $>1$.

To identify the DE probes in transfected cells, we performed Significance Analysis of Microarrays (SAM), a non-parametric statistical test based on a permutation approach specifically implemented for microarray data, imposing a threshold level of False Discovery Rate of $0.05 \%$ (Tusher et al., 2001).

\section{Gene ontology}

DE genes were categorized in gene ontology (GO) classes using the Functional Annotation Clustering method, a bioinformatic tool of the DAVID database (Huang da et al., 2009). Only GO terms with an enrichment score greater than 1.3 and with Pvalue less than 0.05 were considered.

\section{Analysis of discriminant genes}

The list of the best marker genes among transcriptional myofiber classes was obtained applying Prediction Analysis of Microarray (PAM) algorithm (Tibshirani et al., 
2002). PAM utilizes the shrinkage nearest centroid method that does automatic gene selection. Missing values are imputed using a K-nearest neighbor average in gene $\operatorname{space}(\mathrm{K}=10)$.

mRNA q-RT-PCR

\section{Primer design}

qRT-PCR primers (Table S4) were designed by the primer design program Primer3Plus. All primer pairs span intron-exon boundaries. The specificity of the each amplicon was assessed by dissociation curve analysis.

\section{Reverse transcription}

First-strand cDNA was synthetized following SuperScript III (Thermo Fisher Scientific) protocol in a total volume of $30 \mu \mathrm{l}$. Oligo-dT was used for reverse transcription for all the samples.

q-RT-PCR reaction

SYBR Green technology was applied for all the assays with ABI 7500 Standard qRT-PCR System (Thermo Fisher Scientific). The total reaction volume was $10 \mu 1$, including $5 \mu 1$ 2X GoTaq qPCR Master Mix (Promega), $0.6 \mu 1$ of $10 \mu \mathrm{M}$ left primer, $0.6 \mu \mathrm{l}$ of $10 \mu \mathrm{M}$ right primer, $1 \mu \mathrm{l} \mathrm{cDNA}$ template, and $2.8 \mu \mathrm{l}$ of water. Each assay was performed in triplicate. Negative controls without template were added each time. The PCR program started with $2 \mathrm{~min}$. at $95^{\circ} \mathrm{C}$, followed by 40 cycles of two temperature steps $\left(95^{\circ} \mathrm{C}, 15 \mathrm{sec}\right.$; $60^{\circ} \mathrm{C}, 1 \mathrm{~min}$.) and ended with $15 \mathrm{sec}$. at $95^{\circ} \mathrm{C}, 1$ min. at $60^{\circ} \mathrm{C}, 15 \mathrm{sec}$. at $95^{\circ} \mathrm{C}$ and, $15 \mathrm{sec}$. at $60^{\circ} \mathrm{C}$. The last steps of PCR are 
performed to acquire the dissociation curve, validating the specificity of the PCR products. The threshold cycle $(\mathrm{Ct})$ is defined as the fractional cycle number at which the fluorescence exceeds the fixed threshold of 0.2. Three replicates were performed for each reaction. To evaluate differences in mRNA expression, a relative quantification method was chosen where the expression of the mRNA target is standardized by the Txn1 endogenous mRNA, whose level remains essentially constant among different samples (Chemello et al., 2011).

miRNA qRT-PCR

\section{Reverse transcription}

Volume of miRNA population purified from a single myofiber was reduced by speed vacuum concentrator to $6 \mu \mathrm{l}$ and split in two portions. First-strand cDNA was synthetized using the Megaplex RT Primers Rodents Pools A and B (Thermo Fisher Scientific) and the TaqMan MicroRNA Reverse Transcription Kit (Thermo Fisher Scientific) following the corresponding protocol.

\section{Preamplification reaction}

As miRNA amount purified from a single myofiber is very low, specific cDNA targets were amplified to increase the quantity of desired cDNA for expression analysis. Preamplification was performed using TaqMan PreAmp Master Mix Kit (Thermo Fisher Scientific) and Megaplex PreAmp Primers Rodents Pools A and B (Thermo Fisher Scientific) in accordance with the manufacturer's instructions. For each transcriptional signature, pools of three cDNAs from isolated myofibers were prepared for q-RT-PCR experiments. 
$q R T-P C R$

qRT-PCR reactions were performed using TaqMan Universal Master Mix II, no UNG (Thermo Fisher Scientific) and a different TaqMan Small RNA Assay (Thermo Fisher Scientific) for each miRNA analyzed. Three replicates were performed for each reaction. Small nuclear RNA U6 was used as endogenous control, and negative controls without template were added in each plate. Reactions were run in ABI 7500 Standard q-RT-PCR System (Thermo Fisher Scientific) with the following program: $95^{\circ} \mathrm{C}, 10 \mathrm{~min} ; 35$ cycles of $95^{\circ} \mathrm{C} 15 \mathrm{sec}$. and $60^{\circ} \mathrm{C} 1 \mathrm{~min}$. Relative quantification results were obtained using the ExpressionSuite Software (Ver. 1.0, Thermo Fisher Scientific). Pearson's correlation with sequencing data was performed with Excel.

$\underline{\text { NGS of miRNAs purified from single myofibers }}$

\section{Preparation of libraries}

miRNAs libraries preparation partially follows previously set up method for miRNA labeling (Biscontin et al., 2010). Volume of miRNA population purified from a single myofibers was reduced by speed vacuum concentrator to $6.5 \mu 1$. miRNAs were polyadenylated at 3' end using the Poly(A) Tailing Kit (Thermo Fisher Scientific), scaling the volumes of reagents in order to performed the reaction in $10 \mu \mathrm{l}$. Polyadenylated miRNA molecules were precipitated with NaOAc 3 M pH 5.5 (1/10 volume) and absolute ethanol (4 volumes) overnight at $-20^{\circ} \mathrm{C}$ and resuspended in 3.2 $\mu l$ of water. First-strand cDNA was synthetized using SuperScript II (Thermo Fisher Scientific) in a final volume of $10 \mu 1$, including 10 pmol of oligo-dT-Ion P1 Adapter primer (5'-CCTCTCTATGGGCAGTCGGTGATCCTCAGC[dT]20VN-3') and 50 
pmol of SMART primer (5'-CACACACAATTAACCCTCACTAAAggg-3'). ssDNAs having a SMART anchor sequence at the 5'-end was exponentially amplified by 10 PCR cycles performed with Platinum Taq DNA Polymerase High Fidelity (Thermo Fisher Scientific) using as right primer P1 and left primer an A Adapter primer (5'CCATCTCATCCCTGCGTGTCTCCGACTCAG-3') plus a barcode sequence specific for each myofiber type (tS: AAGAG; tI: TACCA; tF: CAGAA) plus SMART primer. A second step of PCR amplification was performed for 10 cycles using as primers A and P1. After each PCR reaction, products were purified twice through GenElute PCR Clean-up columns (Sigma-Aldrich) to eliminate primer-dimers. Library size selection was performed using E-Gel SizeSelect Gels (Thermo Fisher Scientific), according to manufacturer protocol, and recovering dsDNA longer than 120 nucleotides and shorter than 160 .

\section{Sequencing}

Different tagged libraries were pooled together and used for emulsion PCR. Samples were loaded on the Ion OneTouch System (Thermo Fisher Scientific) according to manufacturer specifications and template-positive beads were purified through the Ion OneTouch ES (Thermo Fisher Scientific). Enriched beads were used to load 3 Ion PGM Chips (Thermo Fisher Scientific) and sequencing runs were performed using Ion PGM Sequencer (Thermo Fisher Scientific) according to manufacturer's protocol.

\section{Sequencing data processing}

Raw reads were trimmed and cleaned-up using an in-house developed program that: a) identifies the barcodes of the different myofiber types; b) removes adapters; c) keeps only the reads longer than 18 nucleotides. The expression of miRNAs was 
quantized using miRDeep software (Friedlander et al., 2008). The processed reads were mapped to the known mouse miRNA precursors from miRBase database (Ver. 19) using the mapper module of miRDeep with default values. Basically in this process equal reads are counted and collapse. Reads that mapped more than 5 times are automatically excluded. Quantize module was used to normalize read counts of mature miRNAs.

\section{Analyses of miRNA families}

Enriched miRNA families analysis was performed on miRNAs obtained from NGS of myofibers using miR Family TargetScan database (Ver. 6.2). Only families with a Pvalue (Benjamini-Hochberg adjusted) lower than 0.05 and at least 2 identified miRNAs were considered statistically significant.

\section{Construction of mRNA-miRNA network}

miRNA targets were identified using miRSVR (August 2010 release) and TargetScan (Ver. 6.2) prediction algorithms. Pearson's correlation between mRNA (from microarray) and miRNA (from sequencing) expression data was performed with Excel (Microsoft Office). Only interactions with a correlation coefficient $<0.3$ were considered anti-correlated. Networks were generated and analyzed using Cytoscape software (Ver. 3.2, (Shannon et al., 2003).

\section{$\underline{\text { miRNA mimics over-expression }}$}

C2C12 myoblasts were cultured in Dulbecco's Modified Eagle Medium with high glucose (Life Technologies) $+10 \%$ Fetal Bovine Serum (Life Technologies) in $10 \mathrm{~cm}$ dishes and split every 2 or 3 days before they reached $70 \%$ of confluence. Cells were 
transfected using Lipofectamine 2000 (Life Technologies) following the manufacturer's instructions with $150 \mathrm{nM}$ of mirVana miRNA mimics or scrambled sequence, purchased from Life Technologies. Transfection efficiency was evaluated with Ambion Silencer FAM-Labeled Negative Control (Life Technologies) and estimated of about $60 \%$ by fluorescence microscopy and flow cytometry. Each transfection experiment was independently repeated at least in triplicate. Results were evaluated after 72 hours of transfection.

Hindlimb mouse muscles were transfected using Invivofectamine 2.0 (Life Technologies). Briefly, miRNA mimics were diluted to $0.5 \mathrm{mg} / \mathrm{ml}$ using complexation buffer, mixed to an equal volume of Invivofectamine, and incubated at $50^{\circ} \mathrm{C}$ for $30 \mathrm{~min}$. Immediately $80 \mu 1$ were injected in hindlimb muscles. Injection was repeated after 3 days and muscles were collected for analyses after one week from the first injection. Each transfection experiment was independently repeated at least in triplicate.

Imaging

\section{Immunostaining}

$1 \times 10^{4} \mathrm{C} 2 \mathrm{C} 12$ myoblasts were grown on $13 \mathrm{~mm}$ round coverslip in $25 \mathrm{mM}$ glucose DMEM or FA oxidation medium (for the complete description see Seahorse analyses) and transfected as indicated. Following 72 hours of incubation, cells were fixed for 15 min. at room temperature with $4 \%$ ice-cold formaldehyde. Mitochondria of cells were stained with previously co-transfection with mito-RFP or with anti-TOM20. For antiTOM20 staining, cells were permeabilized for 10 min. with $0.5 \%$ Triton $\mathrm{X}-100$, blocked for 1 hour with $3 \% \mathrm{BSA}$ in PBS, and then stained overnight at $4^{\circ} \mathrm{C}$ with a 
rabbit polyclonal anti-TOM20 (1:150, Santa Cruz). After washing with PBS, cells were incubated with Alexa Fluor 568 Goat Anti-Rabbit IgG (1:1000, Life Technologies) for 1 hour at room temperature. Coverslips were mounted on glass slides using ProLong Gold Antifade Reagent (Life Technologies). Where indicated, neutral lipids were stained before mounting with 15 min. incubation with BODIPY 493/503 ( $2 \mu \mathrm{g} / \mathrm{ml}$, Life Technologies). Each transfection was replicated independently five times. Images were acquired with a $60 \mathrm{X}$ objective using a confocal spinning-disk microscope (Andromeda iMIC system; TILL Photonics). Z-stacks images of ten randomly chosen fields for each coverslip were acquired and stored for subsequent analysis. Images were processed using ImageJ software. Quantification of the fragmentation index $f$ of mitochondrial networks, defined as the sum of relative fragment areas that individually constitute less than $20 \%$ of the total mitochondrial area, was performed using MitoLoc software (Vowinckel et al., 2015). Statistical analysis was performed with GraphPad Prism software.

\section{Muscle cryosection staining}

Succinate dehydrogenase stain was performed incubating fresh muscle cryosections derived from gastrocnemius for $30 \mathrm{~min}$. as described in (Blanco et al., 1988). Periodic acid-schiff (PAS) staining was performed on cryosections of tibialis anterior following the instructions of PAS staining system (Sigma-Aldrich). Images were acquired with a 10X objective using DMR Leica microscope. Bodipy staining was performed incubating cryosections of tibialis anterior with BODIPY 493/503 (2 $\mu \mathrm{g} / \mathrm{ml}$, Life Technologies) for $20 \mathrm{~min}$. Images were acquired with a $60 \mathrm{X}$ objective using Zeiss LM700 Confocal. Images were processed using ImageJ software. Statistical analysis was performed with GraphPad Prism software. 


\section{Electron microscopy}

Samples were fixed with $2.5 \%$ glutaraldehyde in $0.1 \mathrm{M}$ sodium cacodylate buffer $\mathrm{pH}$ 7.4 for 1 hour at $4^{\circ} \mathrm{C}$, post-fixed with $1 \%$ osmium tetroxide and $1 \%$ in $0.1 \mathrm{M}$ sodium cacodylate buffer for 2 hours at $4^{\circ} \mathrm{C}$. After three water washes, samples were dehydrated in a graded ethanol series and embedded in an epoxy resin (SigmaAldrich). Ultrathin sections $(60-70 \mathrm{~nm})$ were obtained with an Ultrotome V (LKB) ultramicrotome, counterstained with uranyl acetate and lead citrate and viewed with a Tecnai G2 (FEI) transmission electron microscope operating at $100 \mathrm{kV}$. Images were captured with a Veleta (Olympus Soft Imaging System) digital camera.

\section{Measurement of oxygen consumption}

Oxygen consumption rate (OCR) was measured with the XF24 Extracellular Flux Analyzer (Seahorse Bioscience). C2C12 transfected myoblasts were seeded in XF24 cell culture microplates at $6 \times 10^{3}$ cells/well in $0.2 \mathrm{ml}$ of DMEM containing $25 \mathrm{mM}$ glucose and incubated at $37^{\circ} \mathrm{C}$ in $5 \% \mathrm{CO}_{2}$. Experiments were carried out on confluent monolayers after 72 hours. OCR in $25 \mathrm{mM}$ glucose DMEM was initiated by replacing the growth medium with serum-free DMEM glucose. OCR driven by lipid oxidation was initiated by replacing the growth medium with substrate-limited medium (DMEM with $0.5 \mathrm{mM}$ Glucose, $1 \mathrm{mM}$ glutamine, $0.5 \mathrm{mM}$ carnitine and 1\% FBS) 9 hours before the experiment. The medium was replaced with the FA oxidation (FAO) medium (2.5 mM Glucose, $0.5 \mathrm{mM}$ carnitine, $111 \mathrm{mM} \mathrm{NaCl}, 4.7 \mathrm{mM} \mathrm{KCl}, 1.25 \mathrm{mM}$ $\mathrm{CaCl}_{2}, 2 \mathrm{mM} \mathrm{MgSO} 4,1.2 \mathrm{mM} \mathrm{NaH} \mathrm{PO}_{4}$, and $5 \mathrm{mM}$ HEPES) 45 min. prior the assay. OCR driven by lipid oxidation was determined by inhibition of the mitochondrial FA importer CPT-I using $40 \mu \mathrm{M}$ etomoxir added 15 min. before the experiment. Just 
prior to starting the assay XF Palmitate-BSA FAO substrate (Seahorse Bioscience, Part \#102720-100) was added to the wells. A titration with FCCP was performed for each transfection in order to determine the optimal FCCP concentration (i.e., the concentration that stimulates respiration maximally), which was found to be $0.4-0.6-1$ $\mu \mathrm{M}$ for scramble, 0.4-0.6-1 $\mu \mathrm{M}$ for miR-27a, 0.4-0.6-1 $\mu \mathrm{M}$ for miR-142. After OCR baseline was established, a solution containing oligomycin (in $25 \mathrm{mM}$ glucose experiment), FCCP, rotenone or antimycin A were sequentially added to each well to reach final concentrations of $1 \mu \mathrm{g} / \mathrm{ml}$ oligomycin, FCCP as stated above, and $1 \mu \mathrm{M}$ for rotenone and antimycin A. Data are expressed as pmol of $\mathrm{O}_{2}$ per min. per plated cells. At the end of each experiment the medium was removed from each well and the total protein content per well was quantified. miRNA transfections were independently replicates at least three times, and for each one five technical replicates were measured. Statistical analysis was performed with GraphPad Prism software.

\section{$\underline{\text { Lipidomic analysis }}$}

\section{LC-MS Measurements}

Lipids were extracted from transfected cells using a solution 2:1 chloroform:methanol $0.005 \%$ BHT. The chloroformic extracts were taken, dried using a rotary evaporator, and dissolved in $150 \mu \mathrm{l}$ HPLC-grade methanol (Sigma-Aldrich). MS measurements were carried out in both positive and negative ion mode, using a Bruker Esquire-LC quadrupole Ion-Trap mass spectrometer equipped with an electrospray source (Bruker Optik GmbH, Ettlingen, Germany). The spectrometer was coupled to an HPLC (Hewlett-Packard Model 1100 Series) to chromatographically resolve the analytes prior to their mass detection. The chromatographic separation was obtained using a 
Zorbax Eclipse XDB-C18 column (150 x 4.6 mm I.D., particle size: $3.5 \mu \mathrm{m}$; Agilent Technologies). The mobile phase consisted of solvent A, 70:30 methanol:water with $12 \mathrm{mM}$ ammonium acetate, and solvent $\mathrm{B}, 100 \%$ methanol with $12 \mathrm{mM}$ ammonium acetate. The elution program started from $35 \% \mathrm{~B}$, reached $100 \% \mathrm{~B}$ in $40 \mathrm{~min}$, and kept this composition for $50 \mathrm{~min}$., at the steady flow rate of $0.8 \mathrm{~mL} / \mathrm{min}$. For the mass detection, the instrumental parameters were the following: scan range 100-1200 m/z with a frequency of $13000 \mathrm{~m} / \mathrm{z} \mathrm{s}^{-1}$, nitrogen pressure of $35 \mathrm{psi}$, temperature of $300^{\circ} \mathrm{C}$, and high voltage capillary of either $4000 \mathrm{~V}$ (positive ionization mode) or $-4000 \mathrm{~V}$ (negative mode). The injection volume was $10 \mu l$.

\section{Data Analysis}

The lipid quantitation was performed by integration of the extracted ion chromatograms (XIC), achieved through the proprietary software Bruker Daltonics esquireLC 4.5. Overall, the following lipid classes were detected: lysophosphatidylcholines (LysoPC), sphingomyelins (SM), phosphatidylcholines (PC), phosphatidylethanolamines (PE), phosphatidylinositols (PI), diglycerides (DAG), triglycerides (TAG), cholesteryl-esters (CE) and cholesterol. In order to compare the results across samples, the signal areas were normalized to the number of cells relative to each sample, which were therefore used as a reference for the quantitation. Experiments were independently repeat in quadruplicate. Statistical analysis was performed with GraphPad Prism software.

\section{Luciferase assay}

Myoblasts were transfected with miRNA mimics and $100 \mathrm{pg} / \mu \mathrm{l}$ of pmirGLO DualLuciferase miRNA Target Expression Vector (Promega) containing the target 
sequence or a control sequence. Assays were performed using the Dual-Luciferase Reporter Assay (Promega), measuring firefly and renilla luciferase activities with Turner Designs TD-20/20 Luminometer (DLReady). miRNA transfections were independently replicates at least three times. Statistical analysis was performed with GraphPad Prism software.

\section{$\underline{\text { Glycogen assay }}$}

Glycogen content in transfected cells was measured using the Glycogen assay colorimetric kit (Abcam) following the manufacturer's instructions. Results derive from the sum of six independent experiments. Statistical analysis was performed with GraphPad Prism software.

\section{$\underline{\text { Flow cytometry }}$}

Transfected C2C12 myoblasts were permeabilized and stained with BODIPY 493/503

(2 $\mu \mathrm{g} / \mathrm{ml}$, Life Technologies) for $15 \mathrm{~min}$. After two PBS washes, cells were transferred in FACS conical tubes and flow cytometry was performed using a BD FACSCalibur platform (Becton Dickinson). Percentage of positive cells was calculated using mock-transfected $\mathrm{C} 2 \mathrm{C} 12$ as a negative control. Results derive from the sum of six independent experiments. Statistical analysis was performed with GraphPad Prism software. 


\section{SUPPLEMENTAL INFORMATION}

\section{Figure S1: Single myofiber isolation and characterization.}

Myofibers were dissociated from soleus and EDL muscles of wild-type CD1 male mice at 3 months of age (Charles River, weight: 33 - $35 \mathrm{~g}$ ). Soleus muscles were collected from mice numbered from 1 to 13 and EDL from mice numbered from 14 to 22. Muscles were separately moved in $1 \mathrm{ml}$ high-glucose DMEM containing type I collagenase. Incubation proceeded for $45 \mathrm{~min}$. at $37^{\circ} \mathrm{C}$. The collagenase-treated muscles were rinsed and single myofibers were liberated by gentle physical trituration with a wide-mouth plastic Pasteur pipette. Intact, non-contracted, and well-isolated myofibers were picked under stereomicroscope and washed. We collected 100 myofibers from soleus muscles and the same number from EDL muscles. About onethird of each fiber was cut and placed in Laemmli buffer for fiber typing while the remaining part was dissolved in TRIzol Reagent for RNA purification. Fiber typing was performed using SDS-PAGE to identify MyHC protein isoforms. After silver staining, bands of MyHC appeared separated and were identified according to their migration rates. As expected, myofibers from soleus muscles were mainly pure 1, 2A, $2 \mathrm{X}$, or hybrid $2 \mathrm{~A} / 2 \mathrm{X}$, whereas myofibers from EDL muscles were mainly pure $2 \mathrm{~B}$ or hybrid 2X/2B. For RNA analysis, we selected 60 myofibers, 10 for each most common myofiber types (biological replicates). RNA was amplified and labeled for microarray hybridization.

\section{Figure S2: Characteristics of myofiber transcriptional signatures.}

(A) PAM test was used for showing the relationship between misclassification errors and number of genes used for building a classification model. The plot obtained by 
running PAM shows that, using a threshold value of 13.3, the expression of 5 genes (dotted line, Myh4, Myh7, Tpm3, Myl3, Actn3) can discriminate among fiber types with complete accuracy. The cross-validation error rate increases when too few or too many genes are involved in this prediction model. Number of genes is indicated at the top. (B) The best genetic markers for the classification of mouse myofibers at transcriptional level, with the assignment of specific scores for soleus or EDL muscles. The higher is the absolute value, the stronger is the classification power of the marker. For each marker, PAM score could be positive (highly expressed in a specific signature) or negative (low or not expressed in a specific signature). Myh2 was arbitrary added to the 5 genes identified by PAM analysis as the best marker of tI myofibers. (C) Expression levels of these genes were measured by qRT-PCR in 15 novel and unclassified myofibers isolated from soleus and 8 from EDL from 3 different mice $(\mathrm{S}=$ soleus, $\mathrm{E}=\mathrm{EDL}$; the first number after the underscore indicates the different mice; the second number, after the dot, indicates the different myofiber). Heat-map represents the normalized expression level against the reference gene Txn1: blue color means low expression, red color high expression, and grey color that the gene expression value was below the threshold of detection. Myofibers and genes were hierarchically clustered using the Euclidean distance and complete linkage methods. This analysis is sufficient to properly classify a myofiber in one of the three transcriptional signatures. At least two myofibers per transcriptional signature were used for miRNAs sequencing (SQ), and three myofibers for miRNAs qRT-PCR validation (RT). (D) K-means clusterization grouped the 1,936 probes with similar expression patterns among the three transcriptional myofiber signatures in 8 different clusters. Expression values (arbitrary units, A.U.) of each probe were separately mediated for the different transcriptional signatures and plotted in the bar graph. The 
numbers of probes of each cluster are indicated in bold, error bars are SEM. Except for cluster 8, each probe cluster shows a preferential expression in one or two myofiber types $(*$ : adjusted P-value $<0.01$, and difference of means $>1$ ): probes of cluster 1 are more expressed in tS myofibers, clusters 2 and 3 in tS and tI myofibers, cluster 4 in tI myofibers, cluster 5 in tI and tF myofibers, and clusters 6 and 7 in tF myofibers (additional information in Dataset S4).

\section{Figure S3: Workflow for the construction of miRNA libraries from single myofibers.}

From the same isolated myofiber (the picture shows an EDL myofiber, scale bar 250 $\mu \mathrm{m})$, mRNA and miRNA populations were separately purified. mRNA was used for classifying myofibers according to the proposed transcriptional classification (tS, tI, or $\mathrm{tF}$ ) by qRT-PCR. miRNA libraries were constructed and amplified using the Switching Mechanism at 5' End of RNA Template (SMART) technology. Briefly, miRNAs were polyadenylated at 3'-end and then retrotranscribed with a modified anchored oligo(dT) primer. This presents on the 5'-end the P1 primer used for Ion Torrent emulsion PCR. Retrotranscription was performed using an enzyme that maintains terminal transferase activity allowing the association of SMART primer to the 3'-end of the cDNA. Second-strand cDNA and full-length miRNA libraries amplification were performed through PCR reaction. Primer P1 was used as reverse primer while as forward primer we used a sequence composed by (5'-3' direction): the primer A for sequencing, a barcode sequence, and the SMART primer. PCR amplification was stopped after 20 cycles in order to achieve a good level of amplification without reaching the plateau phase. This approach allows a strand specific sequencing. 


\section{Figure S4: miRNome of different myofiber transcriptional types.}

(A) miRNAs identified in myofibers were grouped accordingly to their preferential expression by cluster analysis. Expression data $\operatorname{are} \log _{2}$ of sequencing results normalized as counts of reads per million (+1): blue is for poorly expressed (low, L), red for highly expressed (high, H). (B) The expression levels of 55 miRNAs among the three transcriptional myofiber types were measured by qRT-PCR and compared to NGS measurements. An averaged Pearson's correlation of 0.78 was obtained between the two technologies, corroborating the reliability of NGS results.

\section{Figure S5: Post-transcriptional regulatory networks of myofibers.}

Data obtained from microarray (mRNAs) and NGS (miRNAs) experiments of single isolated myofibers were integrated to generate the post-transcriptional regulatory networks of $\mathrm{tS}$, tI, and $\mathrm{tF}$ myofibers. miRNA targets were identified by TargetScan and/or mirSVR prediction algorithms. Only interactions with a correlation coefficient $<-0.3$ were considered. Networks are composed by 1,130 nodes including 79 miRNAs (triangles) and 1,051 mRNAs (squares): blue is for poorly expressed (low, L), red for highly expressed (high, H). They are connected by 5,968 edges (grey to black lines, with color intensity increase paralleling the increase of the anticorrelation between miRNA and mRNA target).

Figure S6: In vitro mitochondrial fragmentation induced by miR-27a-3p and 142-3p.

(A) Electron microscopy of mitochondria of $\mathrm{C} 2 \mathrm{C} 12$ myoblasts transfected with a scramble sequence as control (Scr), with miR-27a-3p, or with miR-142-3p in medium 
containing glucose (GLUC) or palmitate (PALM) as substrate. Scale bar is $500 \mathrm{~nm}$. (B) Representative fluorescence microscopy images of transfected myoblasts in palmitate medium with different levels of mitochondria fragmentation. Mitochondria were stained with mRFP and imaged by confocal microscopy. Images of each cell were captured at different focal depths and then processed by focal plane merging and convolution. Scale bar is $10 \mu \mathrm{m}$. (C) Representative fluorescence microscopy images of single myoblasts. Mitochondria were stained with anti-TOM20 (green, mitochondria) and BODIPY (red, lipids) and analyzed by confocal microscopy. Scale bar is $10 \mu \mathrm{m}$. (D) Global lipid content of myoblasts transfected with a control scramble sequence or with miR-142, and measured with liquid chromatography-mass spectrometry (LC-MS) technology. Lipid quantification was measured as arbitrary units (A.U.) of signal areas of chromatograms and normalized to the number of cells relative to each sample. Number of independent experiments per condition $n=4$. Error bars are SEM and adjusted P-values of ANOVA statistical test was indicated as follow: $* * *<0.001$, and it is referred in comparison to the control. (E) The figure shows the LC-MS quantification of classes of lipids (SM: sphingomyelin, PE: phosphatidylethanolamine, CE: cholesteryl ester, FFA: free fatty acid, CHOL: cholesterol) that show similar concentrations in myoblasts over-expressing miR-142$3 p$ in comparison to control. Number of independent experiments per condition $n=4$. Error bars are SEM; P-values of t-test are $>0.05$.

\section{Dataset S1: MyHC protein and mRNA content of the 60 myofibers sampled for} microarray experiments.

Myofibers for microarray experiments were dissociated from soleus and EDL muscles taken from different mice. Fiber replicates of the same MyHC type were numbered 
from 1 to 10 and marked with muscle of origin ( $\mathrm{S}$ for soleus, and $\mathrm{E}$ for EDL) and number of the animal. About one-third of each fiber was solubilized in Laemli buffer and used for MyHC protein isoform identification by SDS-PAGE. The relative proportions of MyHC isoforms were determined by the measurement of the brightness-area product (i.e., the product of the area of the band by the average brightness, with the subtraction of local background after black-white inversion). The remaining part of the fiber was immersed in TRIzol Reagent for RNA purification and amplification. Expression levels of Myh genes were measured by microarray experiments. Values correspond to $\log _{2}$ spot fluorescence intensity after data normalization (see Materials and Methods). NA indicates that fluorescence of that specific microarray probe is above the detection limit.

\section{Dataset S2: Microarray probes differentially expressed among myofibers.}

Expression profiles were obtained using the Agilent microarray platform and technology. Values of arbitrary units of intensity were $\log _{2}$ transformed and not available (NA) value was assigned if Found and/or Well Above Background Agilent Feature Extraction flags were not positive. Totally, 1,936 DE Agilent probes were identified: a) performing ANOVA one-way analysis, 1,730 probes with adjusted Bonferroni P-value (based on 1,000 permutations) lower than 0.01 were considered DE. The 6 groups considered in the ANOVA analysis corresponded to the 6 myofiber types identified by MyHC protein isoform classification (10 replicates for each group: MyHC-1, -2A, $-2 \mathrm{X},-2 \mathrm{~B}$, and Hyb-2A/2X, $-2 \mathrm{X} / 2 \mathrm{~B})$; b) manually selected probes not recognized by the statistical test and exclusively expressed in one or two transcriptional signatures. A probe is considered expressed in a particular transcriptional signature if the NA number is lesser than $40 \%$ and not expressed if the 
NA number is more than 75\%. 206 probes satisfied these requirements (sheet "DE probes manually recovered").

\section{Dataset S3: PAM test.}

Prediction Analysis for Microarray (PAM) was applied to microarray data to identify a small set of transcriptional markers useful for a quick myofiber classification (tS, tI, and $\mathrm{tF}$ ). Imposing a threshold of 9.0, the list of the top 29 PAM markers was generated. Each PAM-selected mRNA could be a positive marker if it is expressed only in a particular transcriptional signature (PAM score $>0$, red color) or a negative marker if it is not expressed in a particular transcriptional signature (PAM score $<0$, green color).

\section{Dataset S4: Clusters of differentially expressed probes.}

K-means clustering on genes grouped the 1,936 differentially expressed Agilent probes in 8 different clusters, on the basis of their expression. Probes of cluster $1(\mathrm{n}=$ $114)$ are more expressed in tS myofibers, probes of clusters $2(n=385)$ and $3(n=$ $421)$ in tS and tI myofibers, probes of cluster $4(n=95)$ in tI myofibers, probes of cluster $5(n=240)$ in tI and tF myofibers, and probes of clusters $6(n=305)$ and $7(n$ $=333)$ in $\mathrm{tF}$ myofibers. Probes of cluster $8(\mathrm{n}=43)$ do not show preferential expression in any transcriptional signatures.

\section{Dataset S5: Gene Ontology of genes differentially expressed in the 3 transcriptional myofiber signatures.}

DAVID bioinformatic tool v6.7 (http://david.abcc.ncifcrf.gov/) was used for Gene Ontology (GO) analysis of the differentially expressed Agilent probes that share 
common expression patterns among the 3 transcriptional myofiber signatures (tS, tI, and $\mathrm{tF}$ ). In total, 1,893 unique probes (clusters 1 to 7) were used for the analysis and 1,451 were associated with a DAVID ID. The global set of genes in the mouse genome was used as background. One representative GO term from each functional annotation cluster with an enrichment score cutoff $>1.3$ and P-values $<0.05$ was listed. Overlapping functional clusters were ignored. Gene names for each group were listed.

\section{Dataset S6: miRNA identification from isolated myofibers by NGS.}

miRNA populations purified from single isolated myofibers were amplified following SMART technology protocol and sequenced using Ion Torrent PGM Sequencer. Libraries from miRNAs extracted from different myofibers belonging to the 3 transcriptional signatures were univocally flagged with 3 distinct sequence barcodes, in order to analyze in the same sequencing chip more than one library. miRNA libraries of one $\mathrm{tS}$ and one $\mathrm{tF}$ myofiber were placed and sequenced in a single Ion 314 Chip, and miRNA libraries from other $2 \mathrm{tS}, 2 \mathrm{tI}$, and $2 \mathrm{tF}$ myofibers were located and sequenced in further one Ion 316 Chip and one Ion 318 Chip. Raw reads were trimmed and cleaned using a proprietary program that identifies the barcodes of the different myofibers, removes the adapters, and keeps only the reads longer than 18 nucleotides. This produced $1,464,908$ processed reads for tS, 610,994 for tI, and 943,182 for $\mathrm{tF}$ myofibers. The processed reads were mapped to the known mouse miRNA precursors from miRBase (v. 19), the expression levels were quantized using mirDeep and indicated as counts per millions of reads (CPM). This analysis shows that 81 miRNAs are expressed in $\mathrm{tS}$ myofibers, 84 in tI myofibers, and 75 in $\mathrm{tF}$ 
myofibers, for a total of 137 miRNAs found in at least one myofiber transcriptional type.

\section{Dataset S7: miRNA families expressed in muscle fibers.}

Enriched analysis of miRNA families was performed on myofiber miRNA sequences produced by NGS, using the miR Family TargetScan database (Ver. 6.2). Only families with a P-value (Benjamini-Hochberg adjusted) lower than 0.05 and at least 2 identified miRNAs were considered statistically significant and listed. Families containing known myomiRs are marked in red color.

\section{Dataset S8: miRNA qRT-PCR of single myofibers.}

55 miRNAs identified by NGS were tested by qRT-PCR in the 3 different myofiber types. mRNA and miRNA populations were separately purified: mRNA fractions were used for myofiber transcriptional classification and then miRNAs of three myofibers per transcriptional type ( $\mathrm{tS}$, tI, and $\mathrm{tF}$ ) were used for qRT-PCR experiments. U6 was used as endogenous reference to calculate $\Delta \mathrm{CT}$ values.

\section{Dataset S9: miRNA-mRNA targets network.}

To identify miRNA putative targets, we integrated for the analysis two complementary algorithms: a) TargetScan (version 6.2, release June 2012), that predicts biological targets of miRNAs by searching for the presence of conserved sites that match the seed region; b) mirSVR (release August 2010), a machine learning method able to identify also non-canonical and non-conserved miRNA target sites. In addition, only interactions with a negative Pearson's correlation coefficient $(<$ -0.3) between miRNA and mRNA expressions among the three transcriptional 
signatures were considered. miRNA expression values were reported as $\log _{2}$ of count per million +1 , while mRNA expression values were reported as the mean of $\log _{2}$ of the arbitrary units of microarray intensities. Values of the different probes identifying the same transcript were mediated. Each interaction was predicted by one or both prediction algorithms $(\mathrm{TS}=$ TargetScan, SVR $=$ mirSVR, TS-SVR $=$ both $)$. The sheet "Number of direct edges" reports the number of edges for each node.

\section{Dataset S10: Lipidomic analysis.}

Lipids were extracted from at least half million of myoblasts transfected with a scrambled sequence (Scr, as control), or with miR-142-3p, and measured with LCMS. In order to compare the results across samples, the signal areas were normalized to the number of cells relative to each sample, which were therefore used as a reference for the quantitation $(n=4) .66$ lipids were identified (carbons:double bounds), belonging to 10 different classes. Lyso-PC: lysophosphatidylcholine, SM: sphingomyelin, PC: phosphatidylcholine, PE: phosphatidylethanolamine, PI: phosphatidylinositol, DAG: diacylglycerol, TAG: triacylglycerol, CE: cholesteryl ester, FFA: free fatty acid, CHOL: cholesterol.

\section{Dataset S11: Gene Ontology categories down-regulated in myoblasts over- expressing miR-27a-3p or miR-142-3p.}

As miRNAs repress the expression of their biological targets, Gene Ontology (GO) enrichment analysis of down-regulated genes in myoblasts over-expressing miR-27a$3 p$ or miR-142-3p was performed using the DAVID bioinformatic tool. A single representative GO term from each functional annotation cluster with an enrichment 
bioRxiv preprint doi: https://doi.org/10.1101/288035; this version posted March 23, 2018. The copyright holder for this preprint (which was not certified by peer review) is the author/funder. All rights reserved. No reuse allowed without permission.

score $>1.3$ (the higher, the better) and P-values $<0.05$ (the lower, the better) was listed. Overlapping functional terms were ignored. 


\section{A Fig. 1 Skeletal muscle}

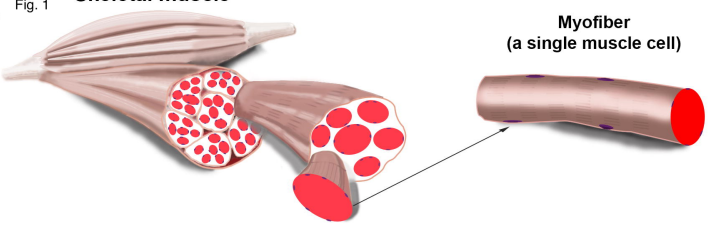

B

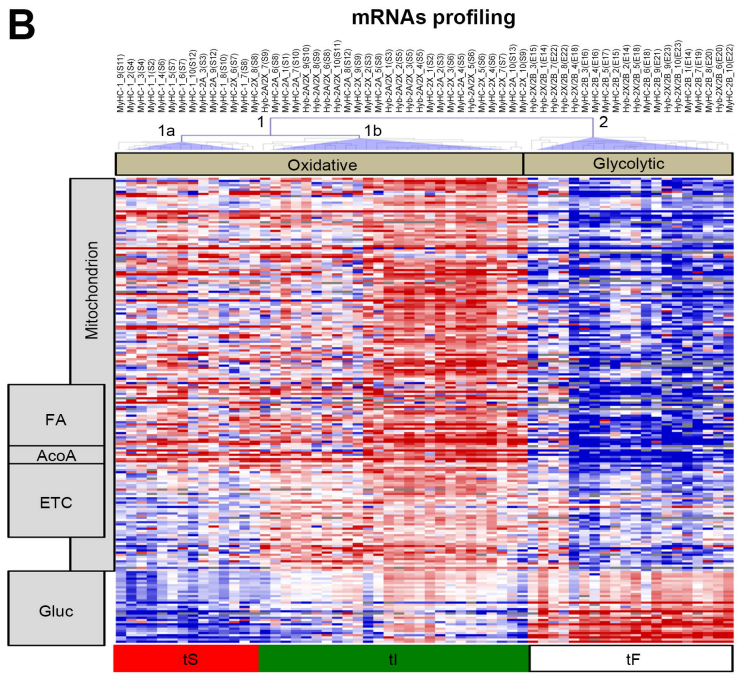

miRNA-mRNA integration

Sub-network 1:

- Mitochondrion

- Myofibril

- Fatty acid metabolic process

- Electron transport chain

- Oxidative phosphorilation

- Acetyl-CoA metabolic

process

- Peroxisome

- Angiogenesis
C

Myofibers classification

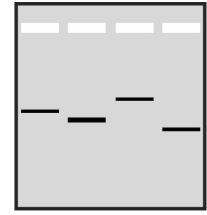

miRNAs profiling
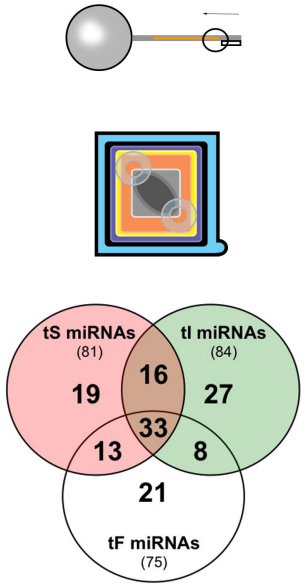

Sub-network 2:

- Muscle contraction

- Glucose metabolic process

- Calcium signaling

- Glutathione transferase activity

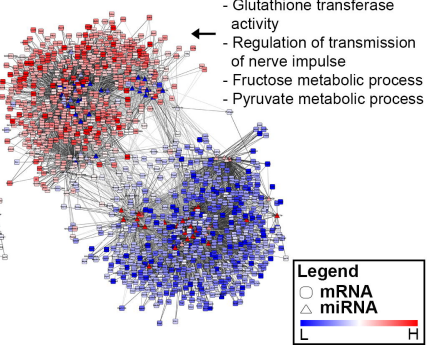


$\mathbf{A}^{\mathrm{Fig} .2}$ B

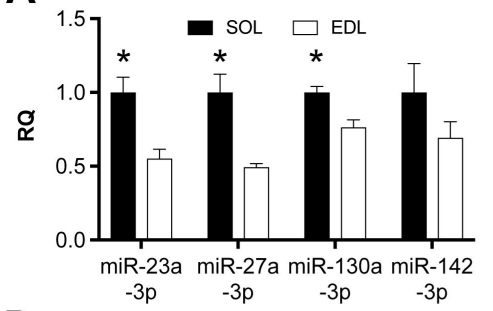

D

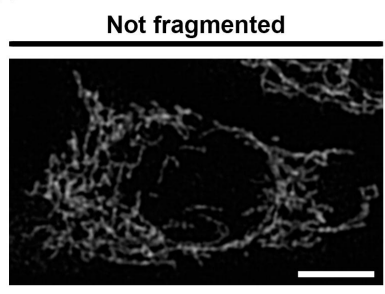

Scr

$\mathbf{F}$

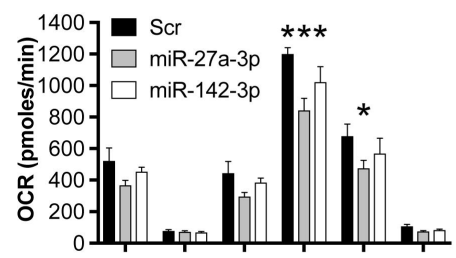

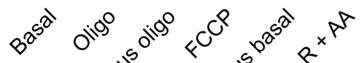

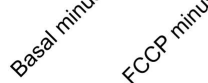

Palmitate medium

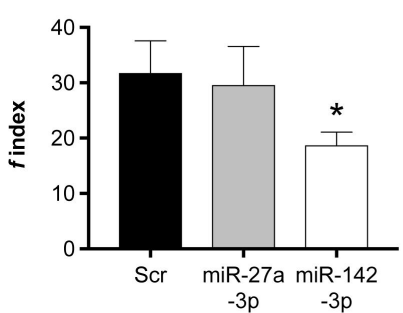

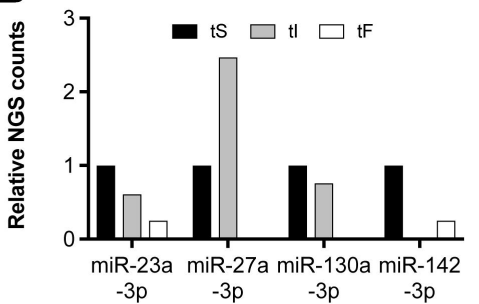

Fragmented

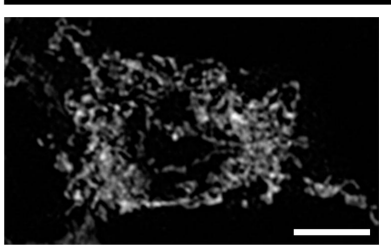

miR-27a-3p

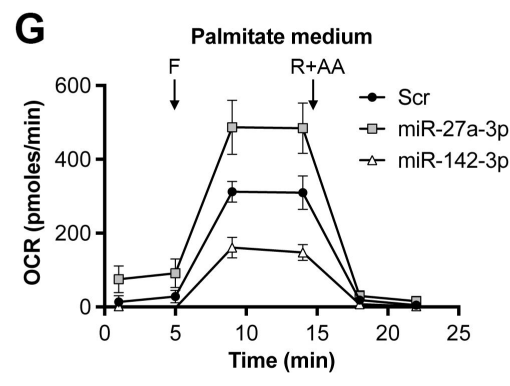

C Glucose medium

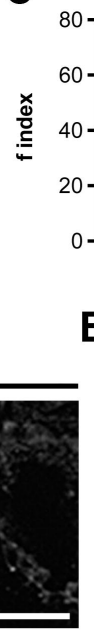

H

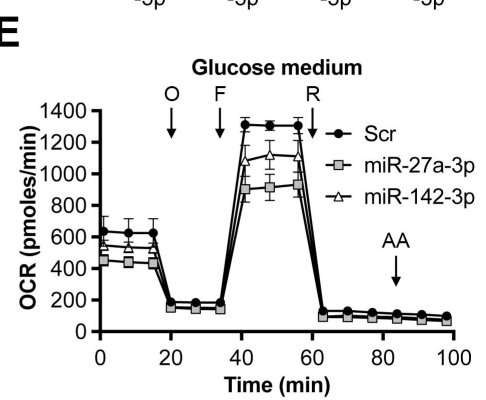

$\begin{array}{ccccc}\text { Scr } & \text { miR-23a } & \text { miR-27a } & \text { miR-130a } & \text { miR-142 } \\ -3 p & -3 p & -3 p & -3 p\end{array}$

E

Palmitate medium
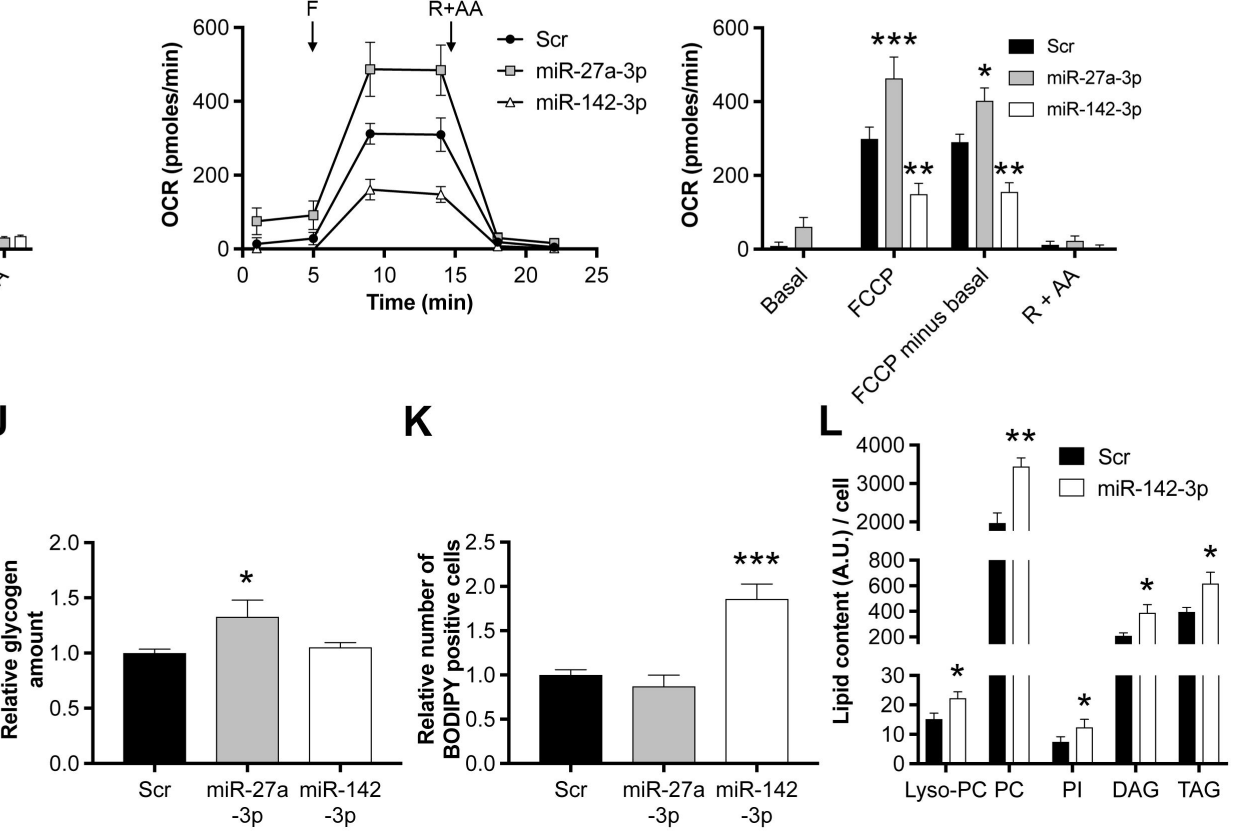

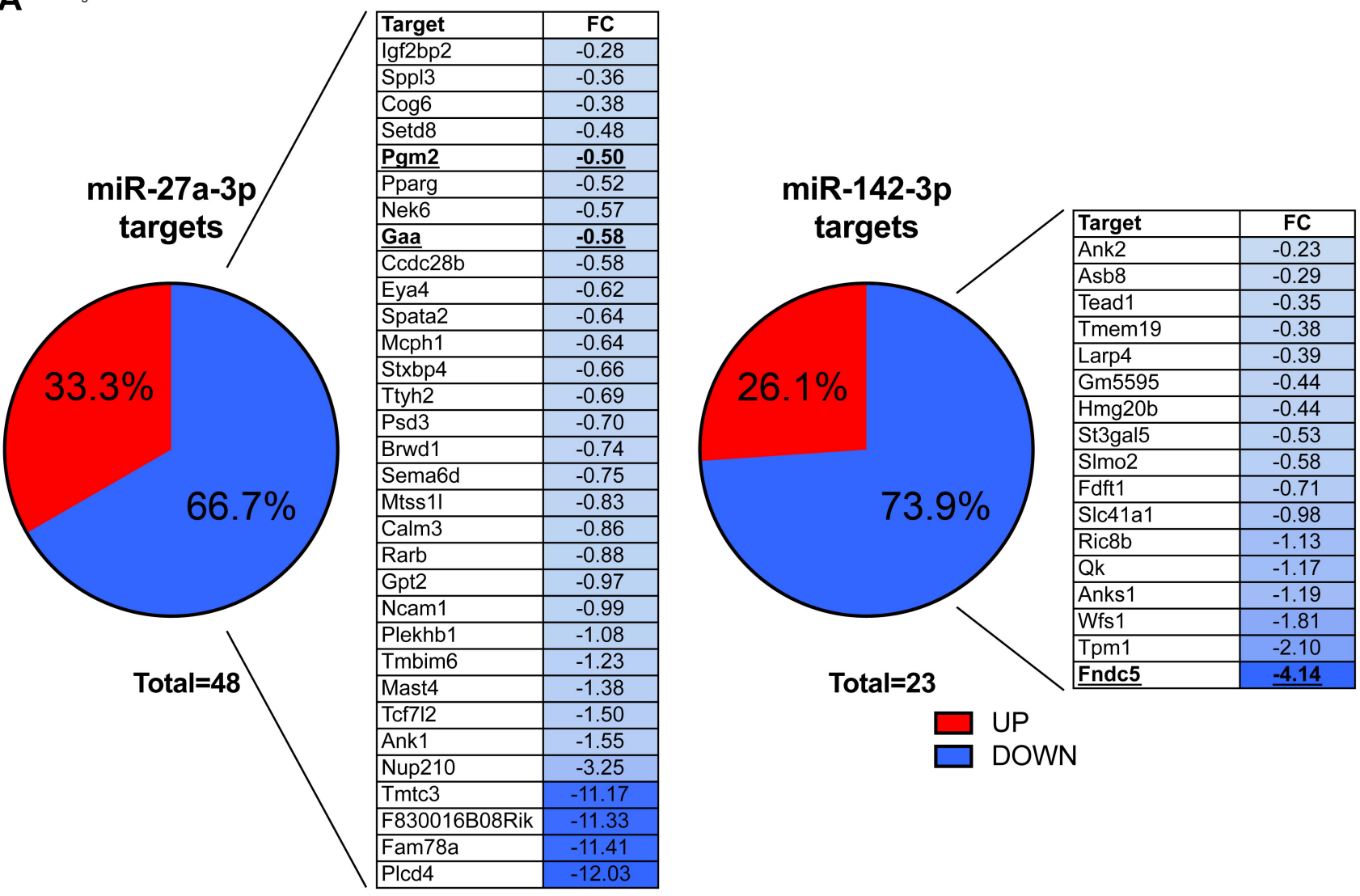

B

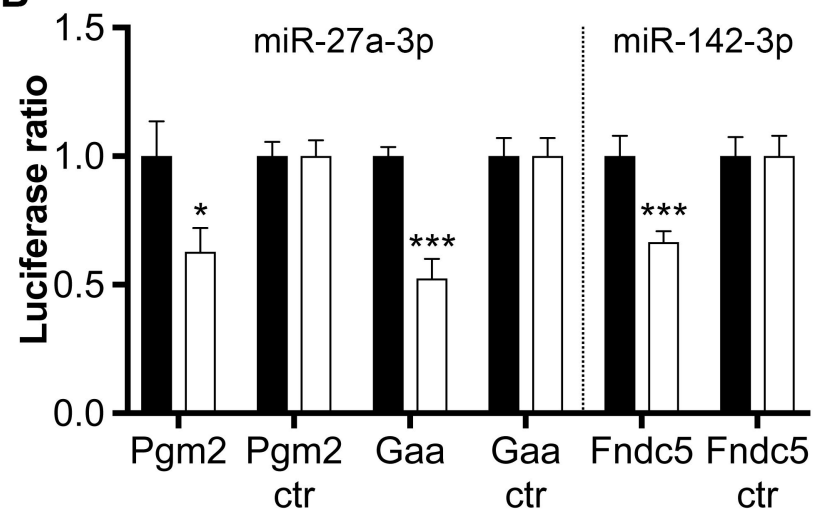

C

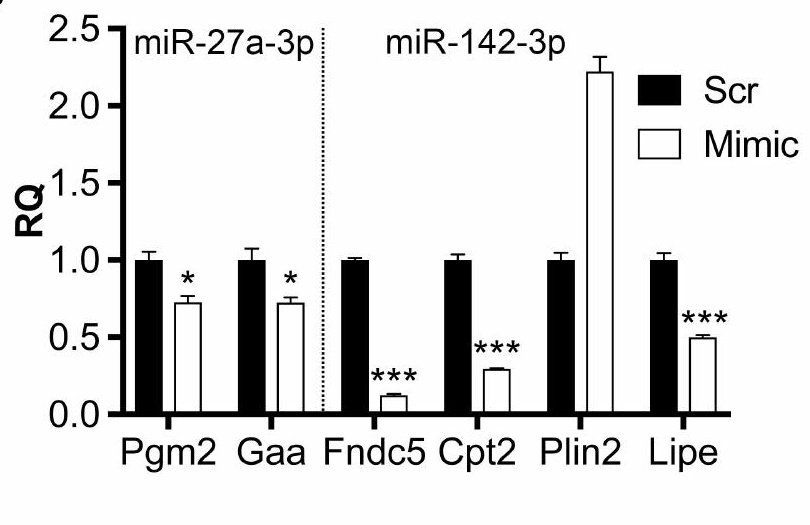




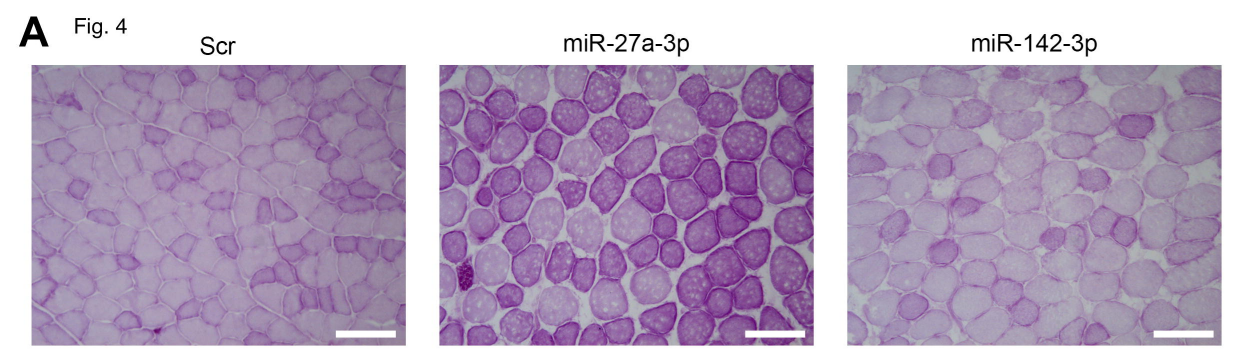

B Glycogen staining

C

LONG

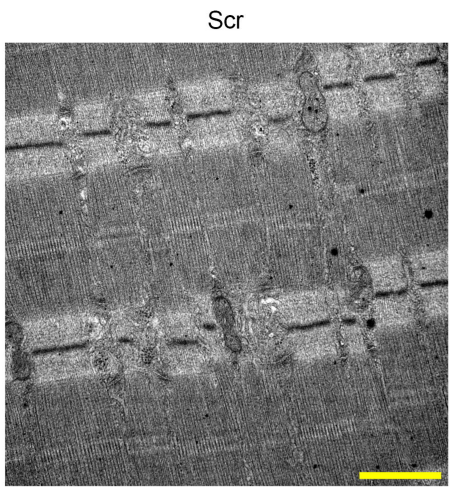

TRANS

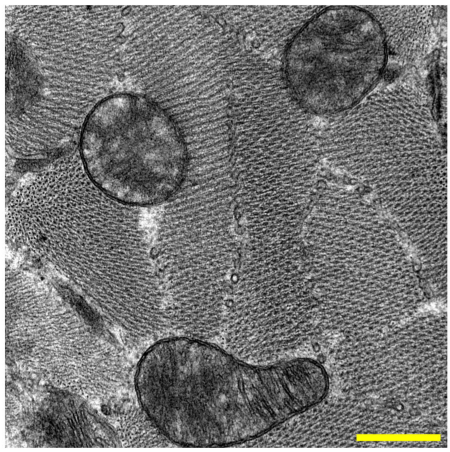

D

Scr

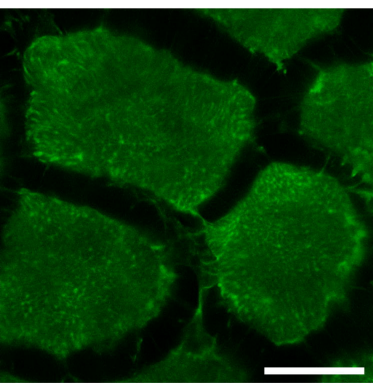

F

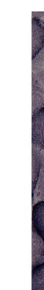

Scr

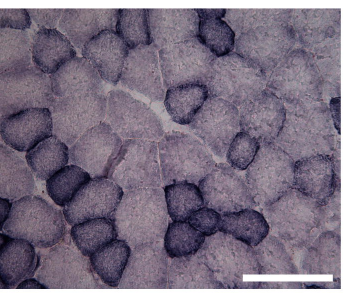

miR-27a-3p

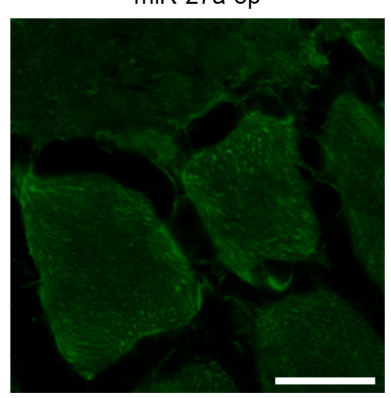

miR-27a-3p

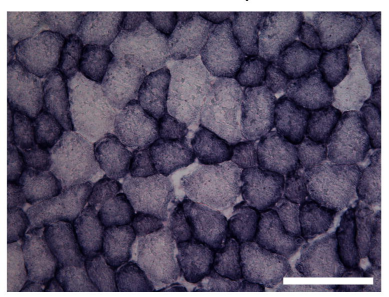

miR-27a-3p
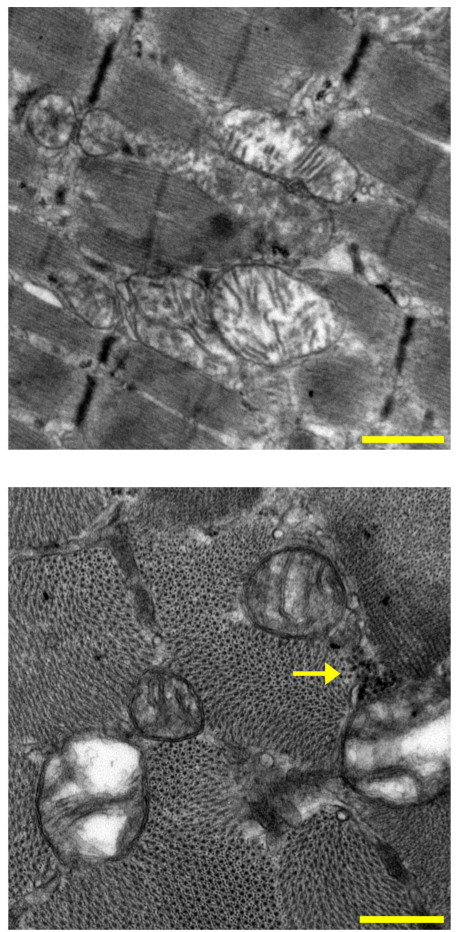

miR-142-3p

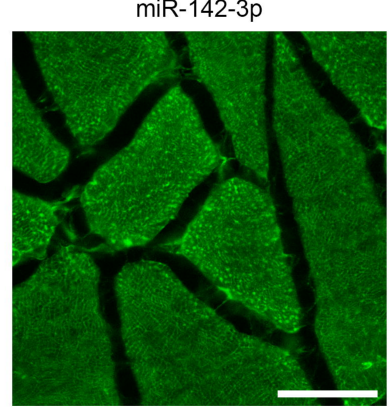

miR-142-3p

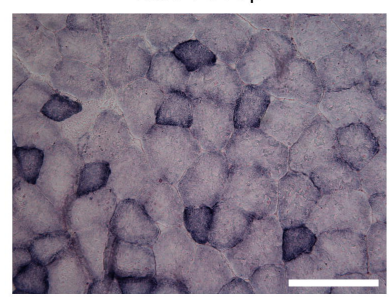

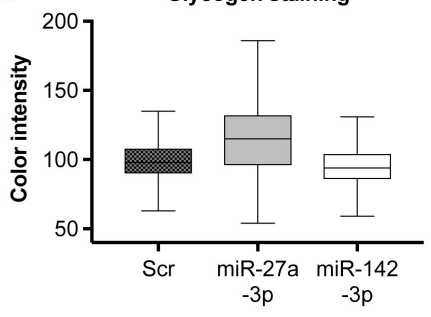

miR-142-3p
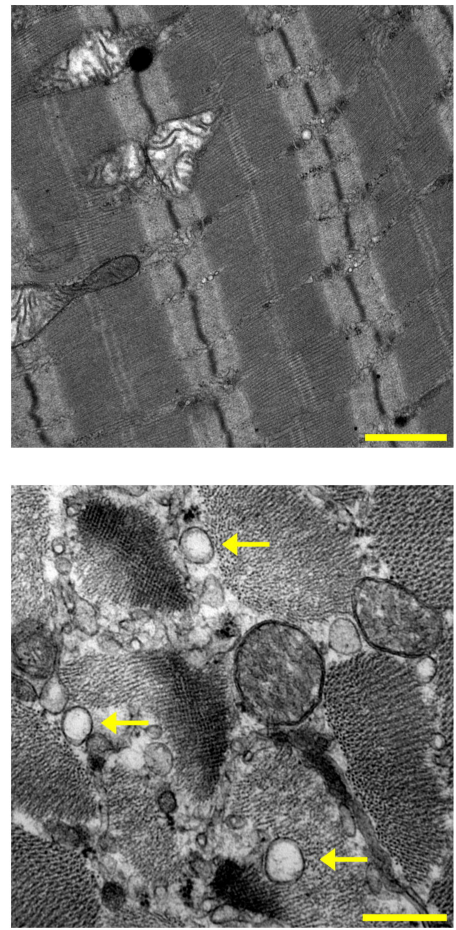

E
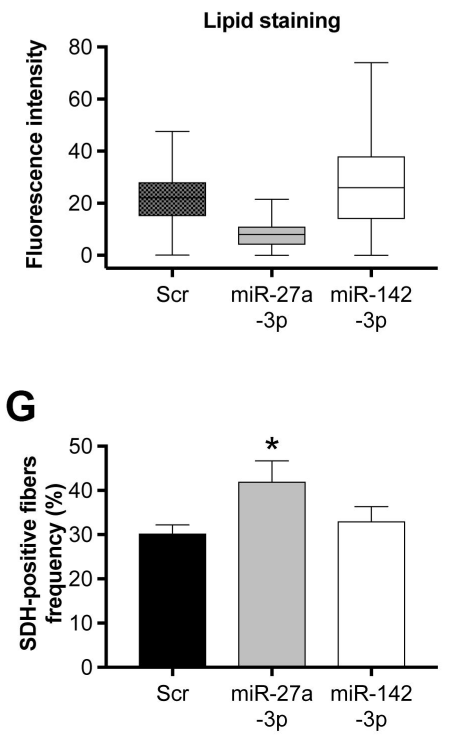
A

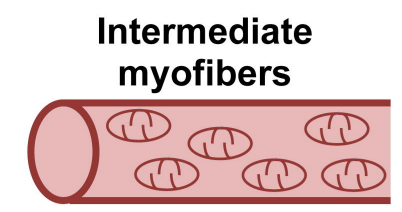

Rich in mitochondria, oxidative metabolism

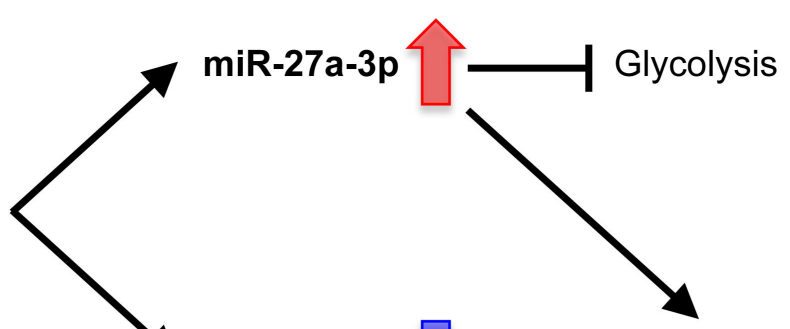

miR-142-3p

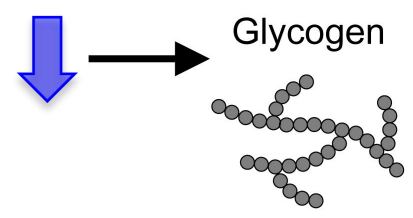

B

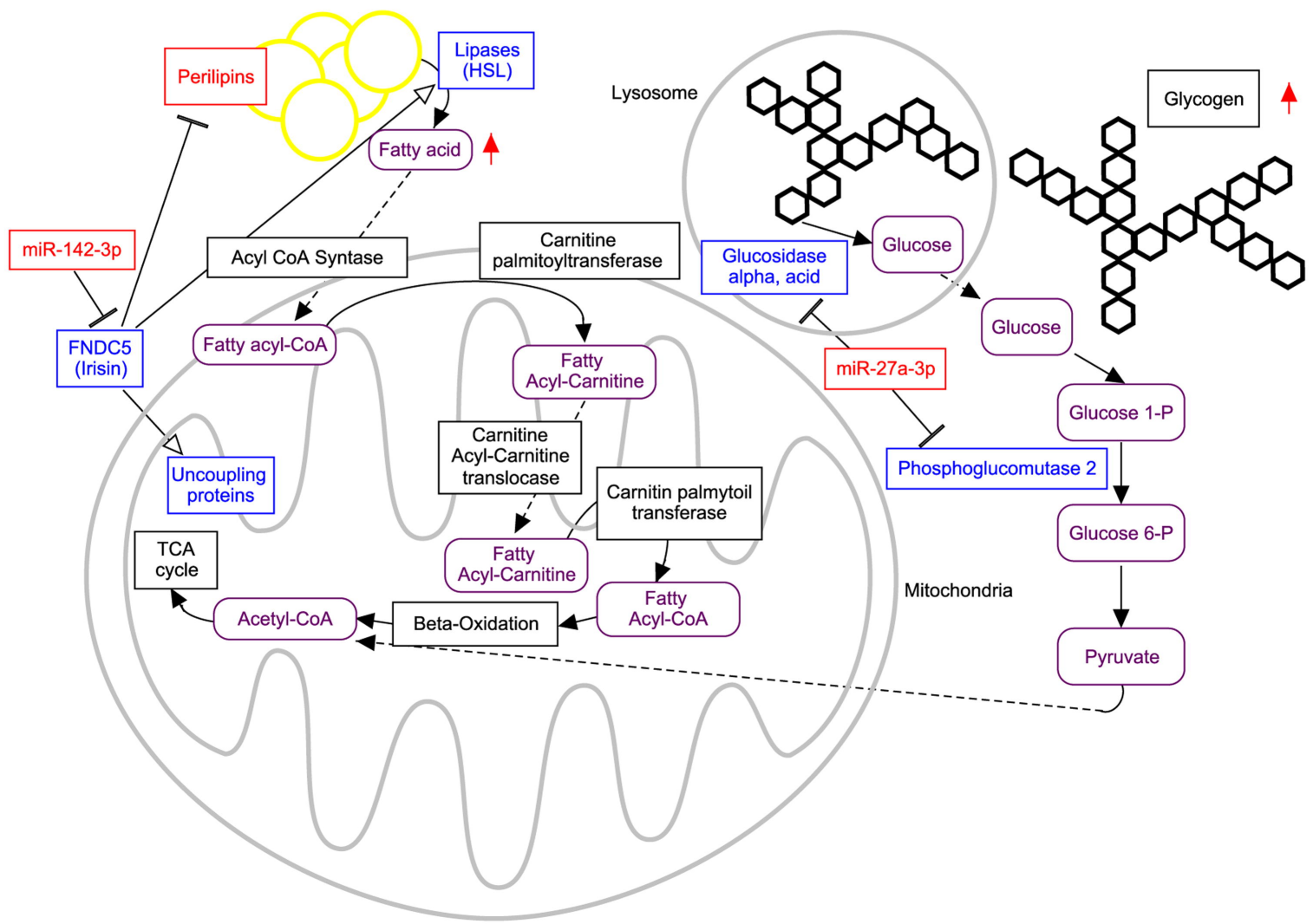




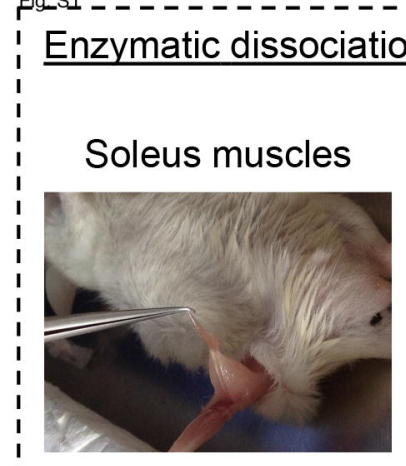

CD1 mice 1-13

(3 months age, 33-35 grams)

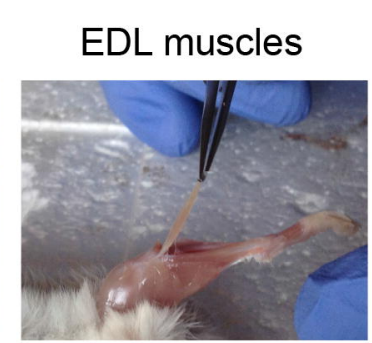

CD1 mice 14-22 (3 months age, 33-35 grams)
Collagenase incubation

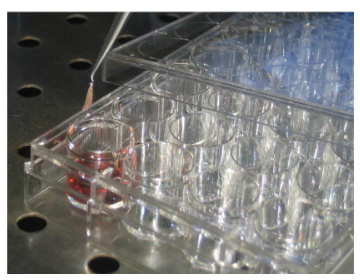

Type I collagenase $10 \mathrm{mg} / \mathrm{ml}$, $37^{\circ} \mathrm{C}, 45 \mathrm{~min}$.

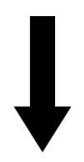

Single myofiber dissociation

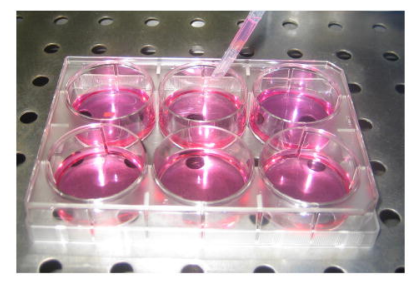

Gentle physical trituration with a wide-mounth plastic

Pasteur pipette.

\section{MyHC isoform classification}

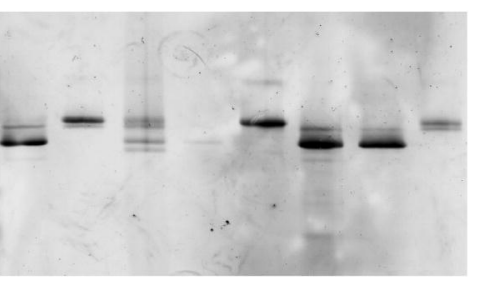

SDS-PAGE gel $4^{\circ} \mathrm{C}$ for $43 \mathrm{~h}$, silver staining

Most abundant myofiber types identified:

- from soleus: pure 1 , $2 \mathrm{~A}, 2 \mathrm{X}$, and hybrid $2 \mathrm{~A} / 2 \mathrm{X}$;

- from EDL: pure 2B, and hybrid $2 \mathrm{X} / 2 \mathrm{~B}$.

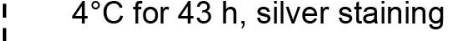

\section{Single myofiber collection}

100 individual myofiber from Soleus 100 individual myofiber from EDL

Each fiber was cut in 2 parts: one for protein classification, the other for microarray experiments.
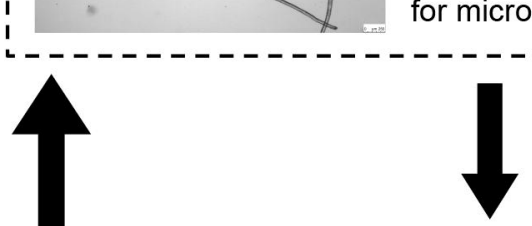

Microarray hydridization

\begin{tabular}{|c|c|c|}
\hline Soleus & EDL & \multirow{5}{*}{$\begin{array}{c}\text { Myofiber } \\
\text { selection } \\
\text { (tot: } 60 \\
\text { fibers) }\end{array}$} \\
\hline $10 \mathrm{MyHC-1}$ & $10 \mathrm{Hyb}-2 \mathrm{X} / 2 \mathrm{~B}$ & \\
\hline $10 \mathrm{MyHC}-2 \mathrm{~A}$ & $10 \mathrm{MyHC-2B}$ & \\
\hline $10 \mathrm{Hyb}-2 \mathrm{~A} / 2 \mathrm{X}$ & & \\
\hline $10 \mathrm{MyHC}-2 \mathrm{X}$ & & \\
\hline
\end{tabular}

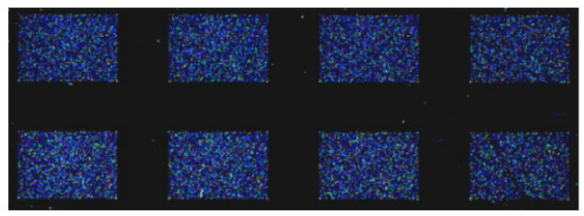

SurePrint G3 Mouse Gene Expression 8x60K microarray platforms (Agilent Technologies) 
$\mathbf{A}{ }^{\mathrm{Fig} . \mathrm{S2}}$

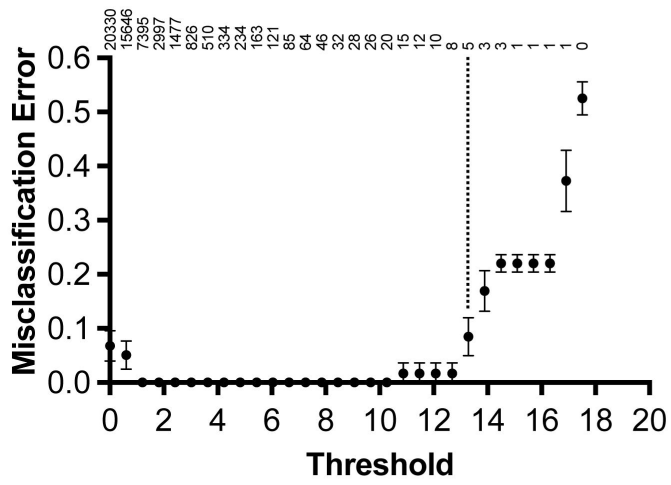

C

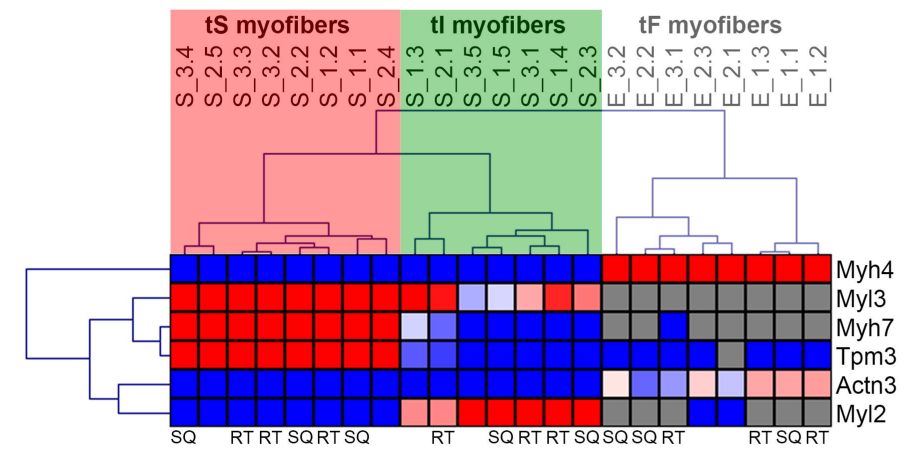

B

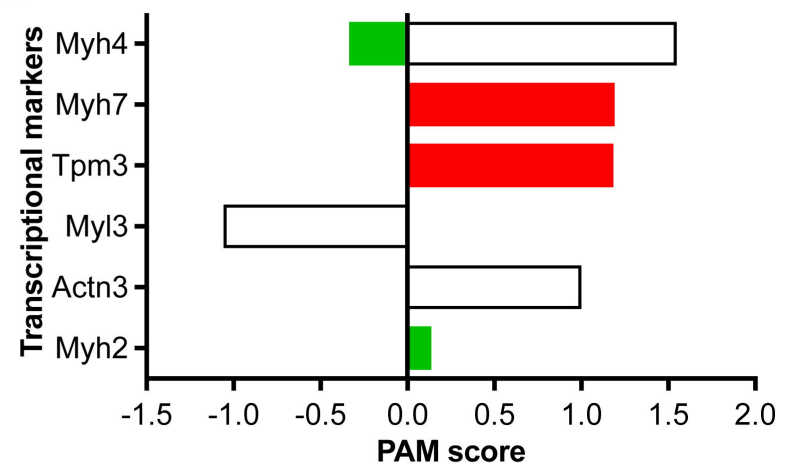

$\square$ tS myofibers $\square$ tl myofibers $\square$ tF myofibers

D

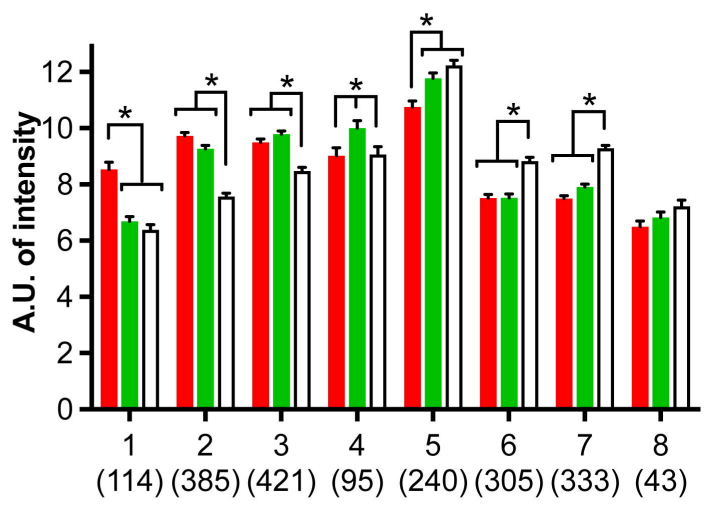


Single myofiber isolated from soleus or EDL mouse muscles.
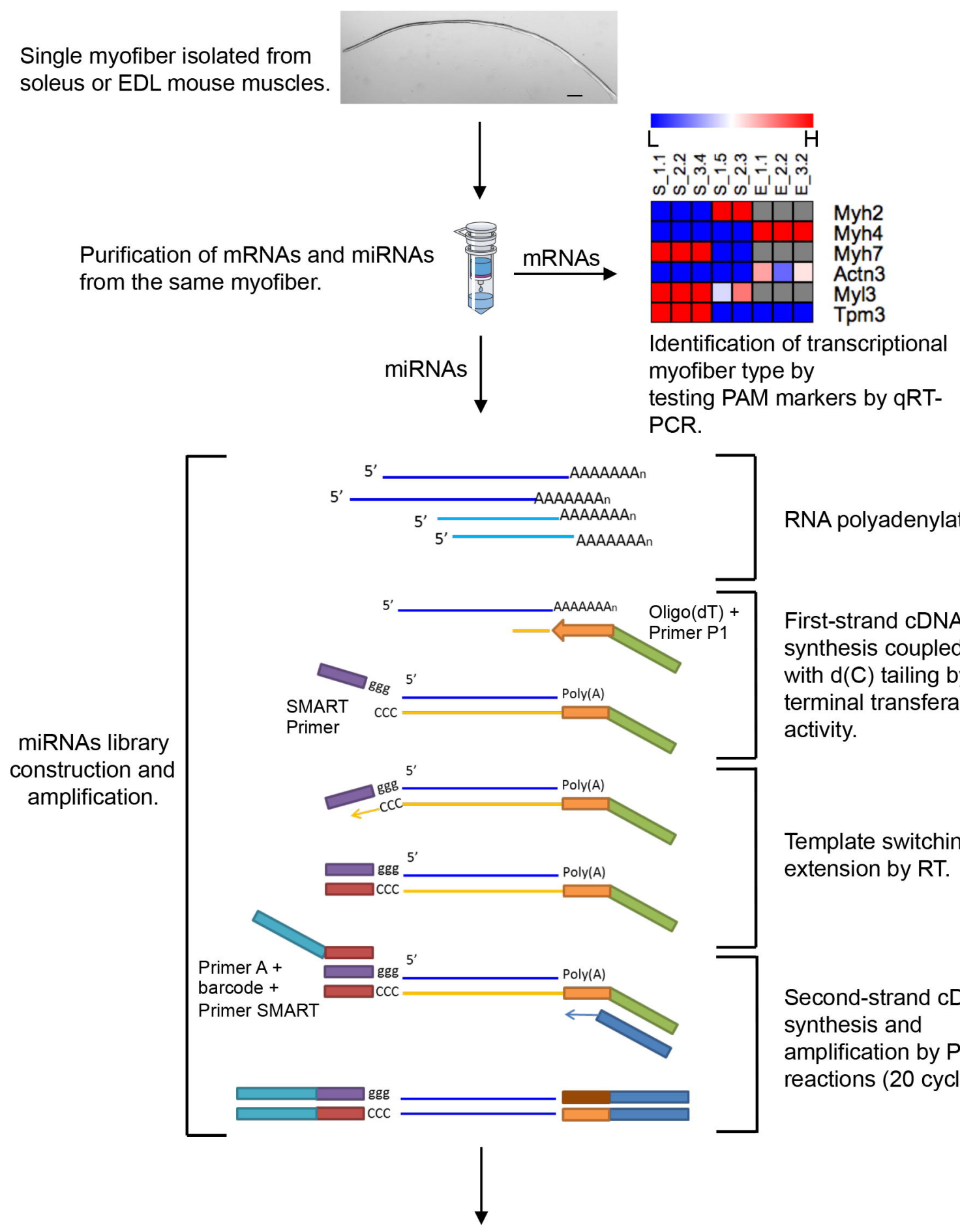

RNA polyadenylation.

First-strand cDNA synthesis coupled with $d(C)$ tailing by $R T$ terminal transferase activity.

Template switching and extension by RT.

Second-strand cDNA synthesis and amplification by PCR reactions (20 cycles). 
$\mathbf{A}^{\mathrm{Fig} . \mathrm{S4}}$
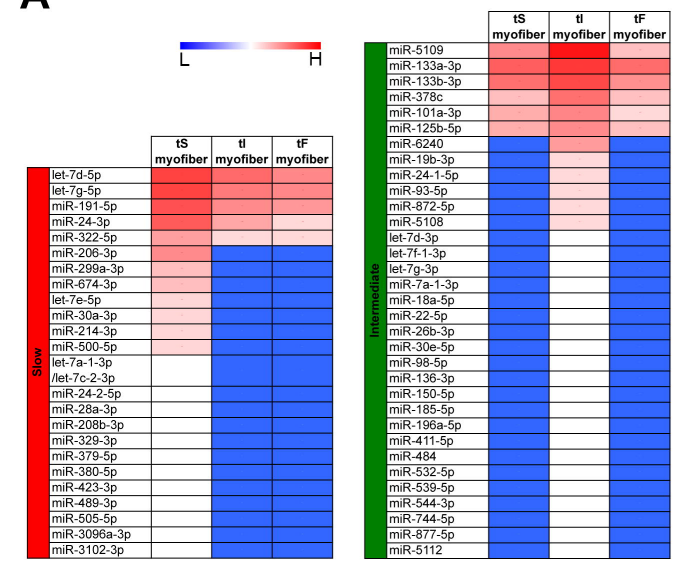
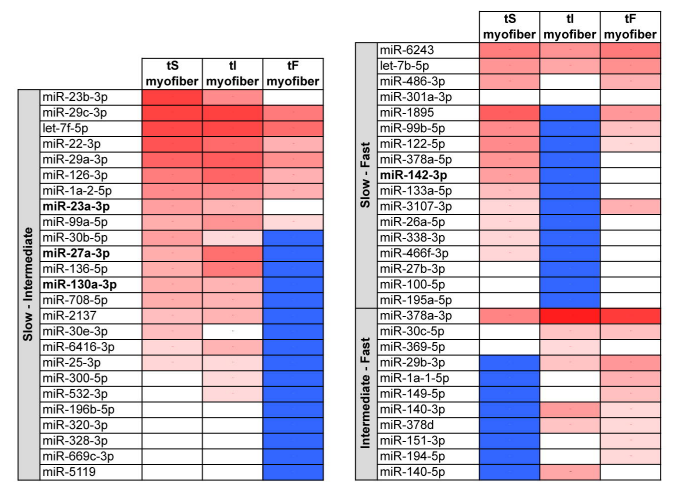

\section{B}

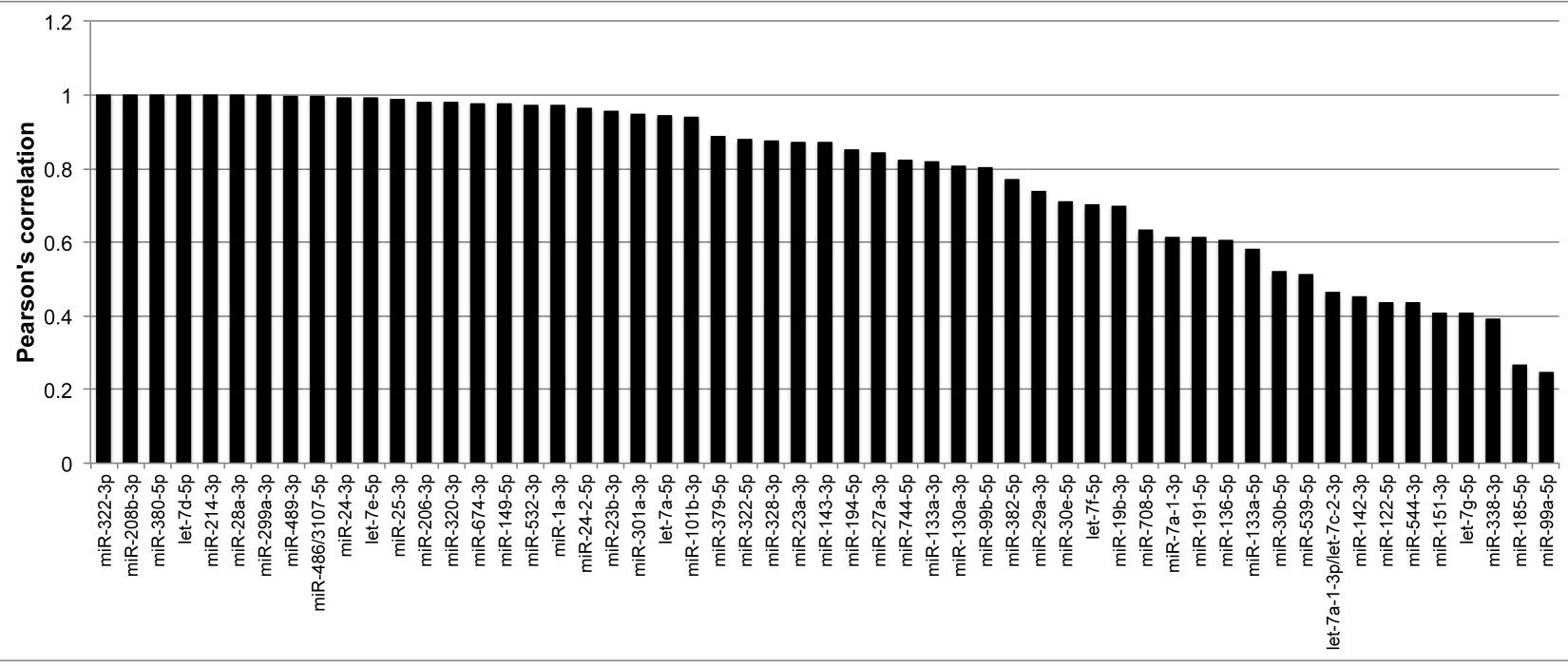




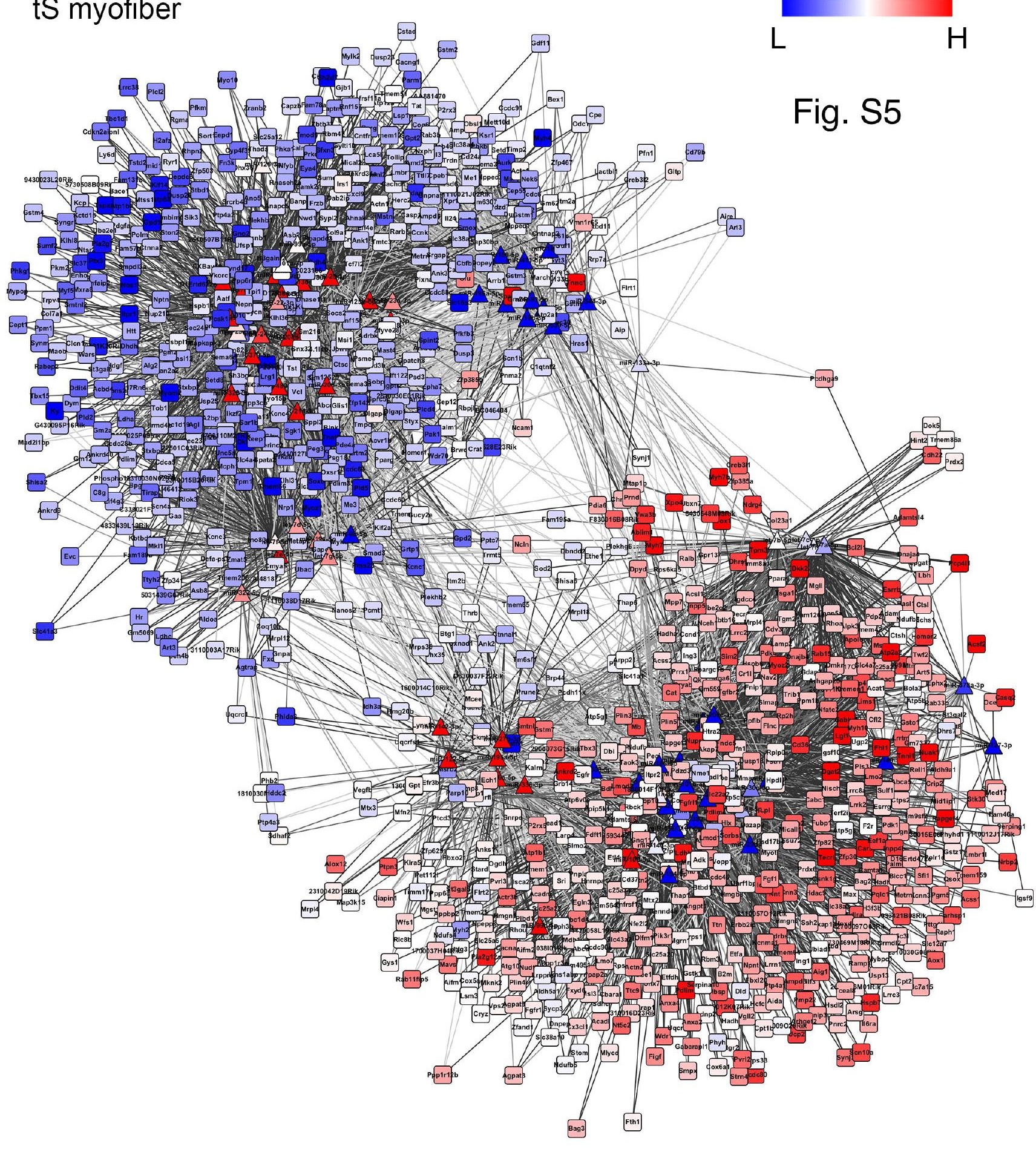

tl myofiber

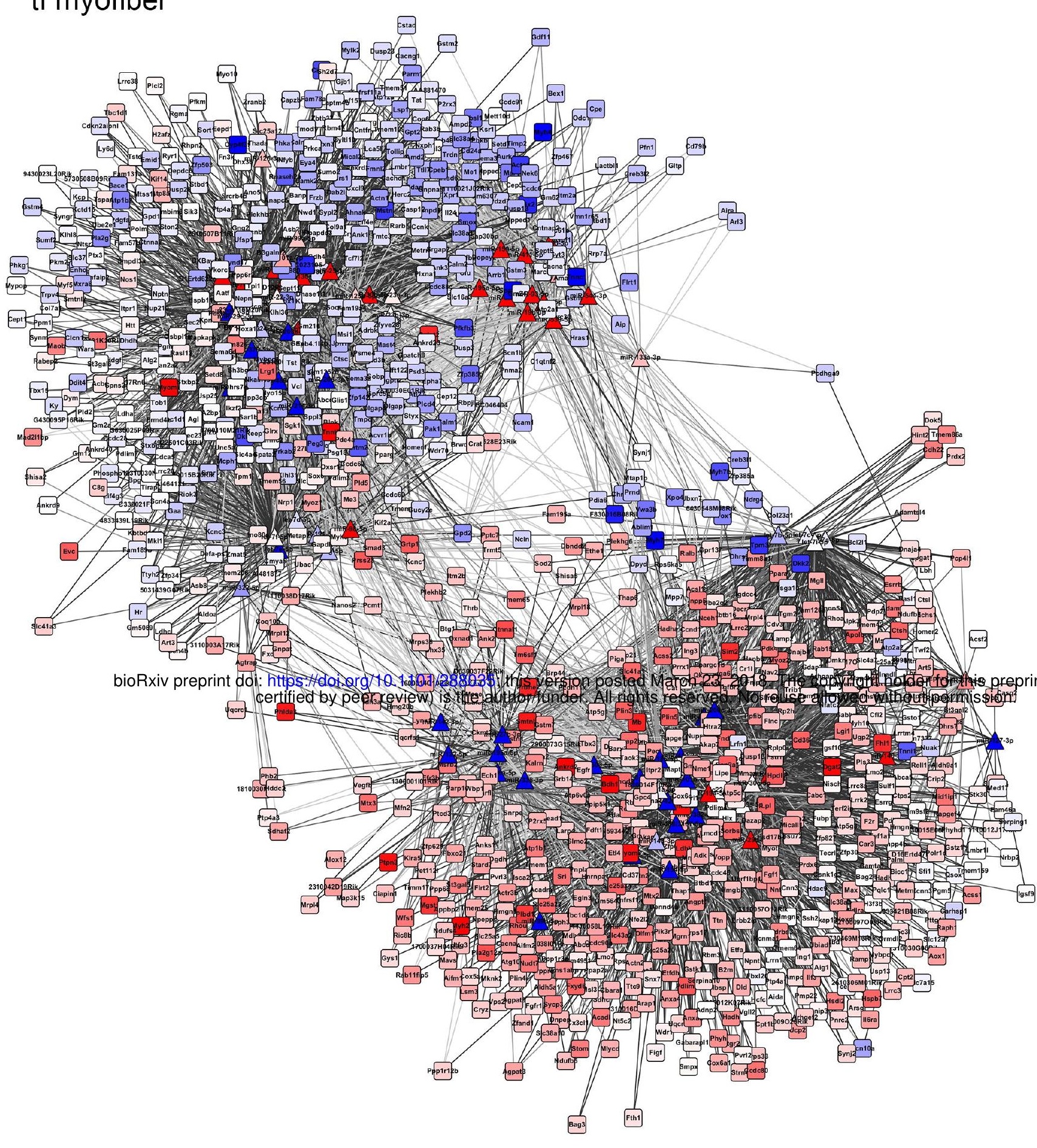

tF myofiber

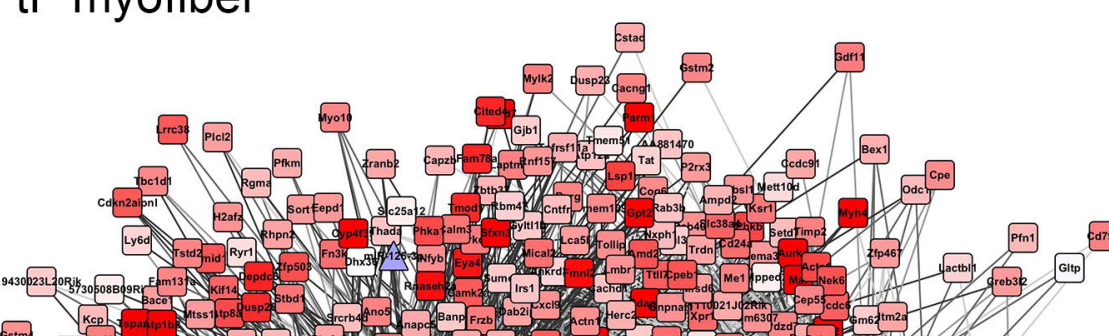

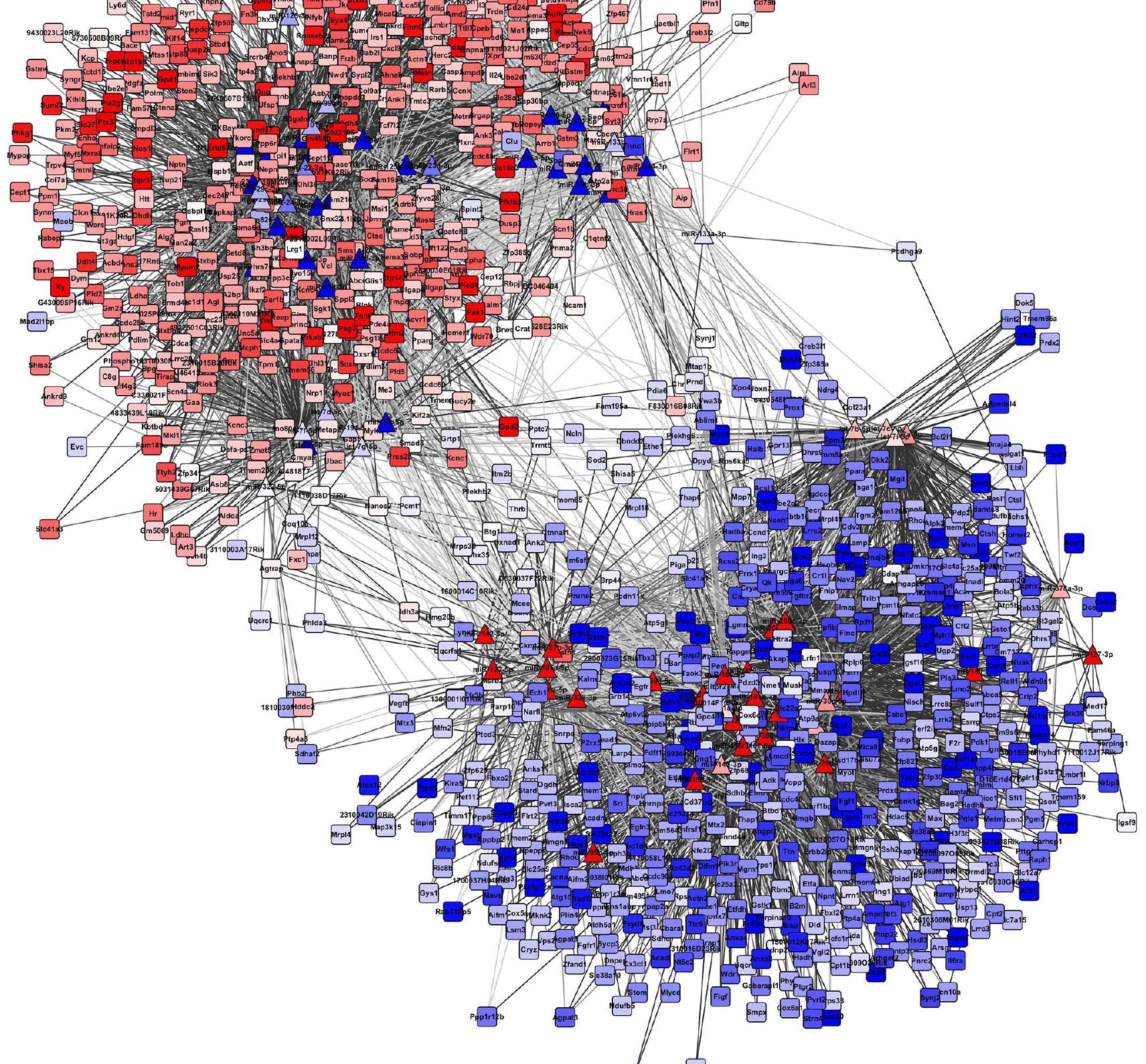


A Fig. S6

certified by peer review) is the author/funder. All rights reserved. No reuse allowed without permission.
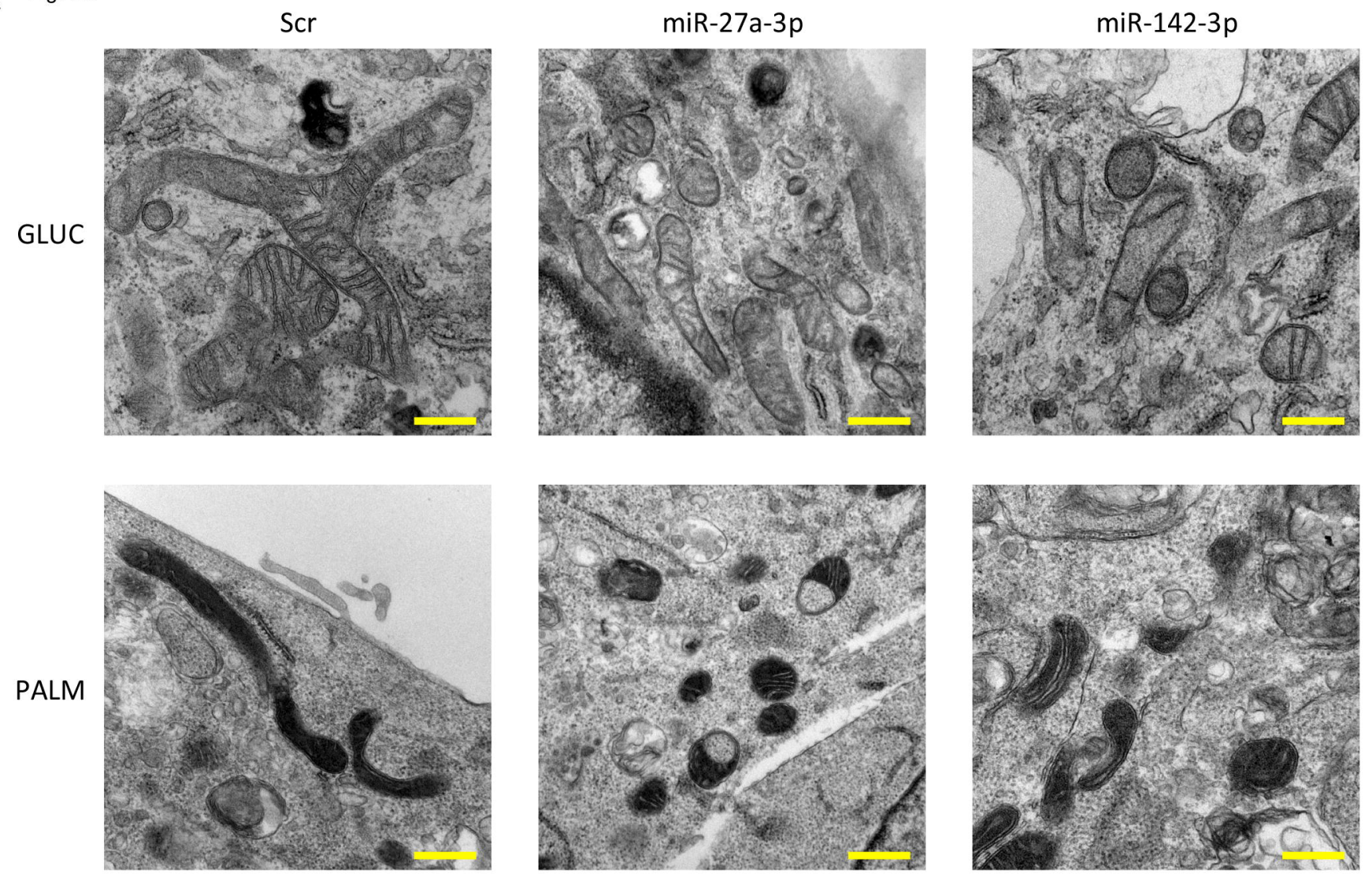

\section{Not fragmented}

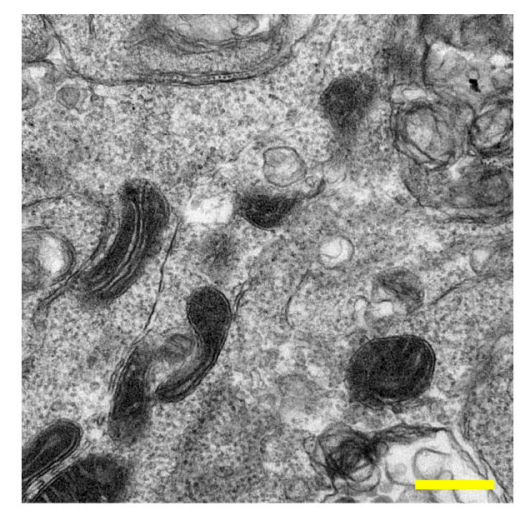

Fragmented
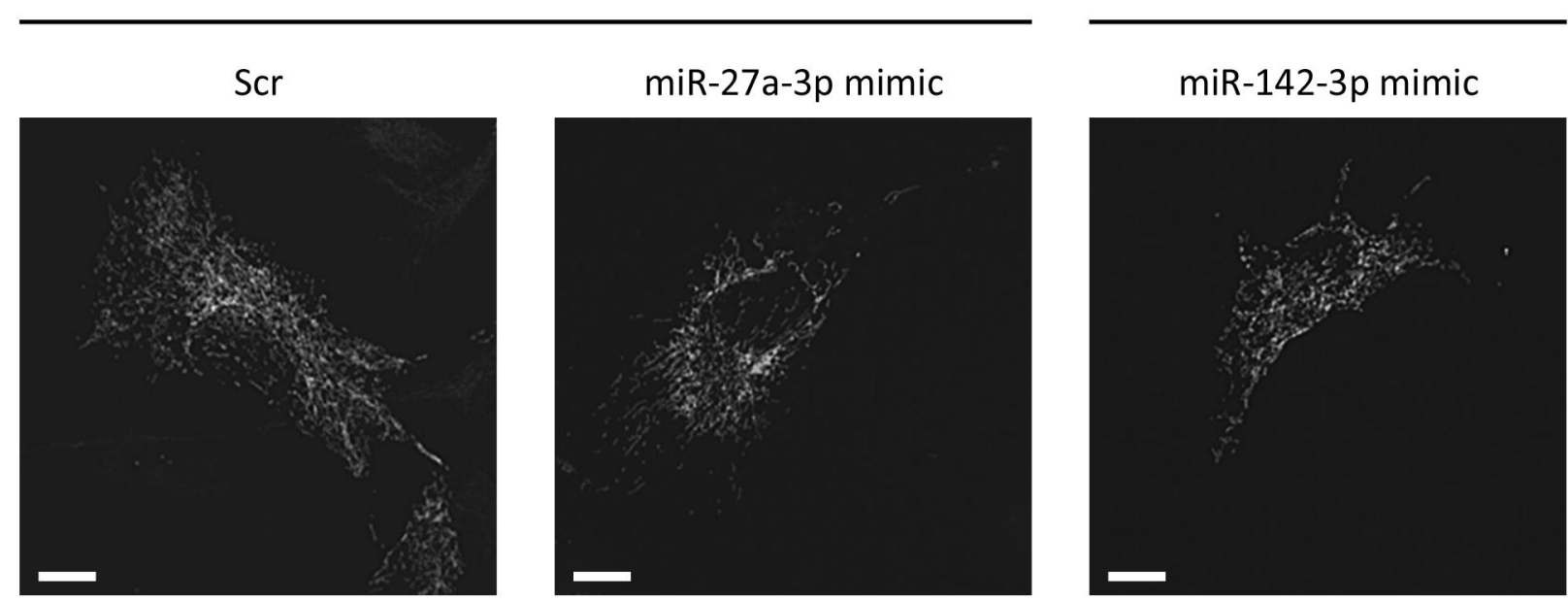

C
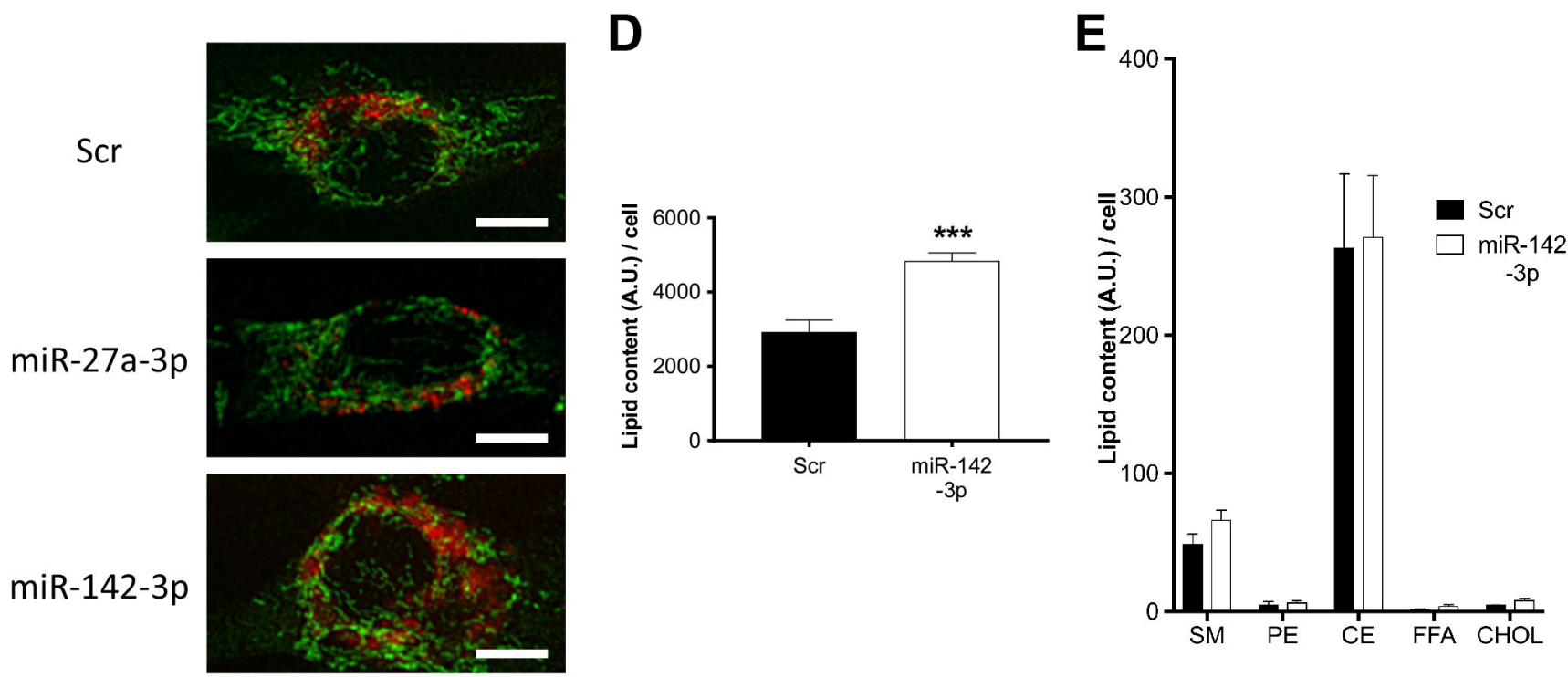